\title{
Analyzing the Combination of Conflicting Belief Functions.
}

\author{
Philippe Smets \\ IRIDIA \\ Université Libre de Bruxelles \\ 50 av. Roosevelt, CP 194-6, 1050 Bruxelles, Belgium \\ psmets@ulb.ac.be \\ http://iridia.ulb.ac.be/ ${ }^{\sim}$ psmets
}

March 31, 2005

\begin{abstract}
We consider uncertain data which uncertainty is represented by belief functions and that must be combined. The result of the combination of the belief functions can be partially conflictual. Initially Shafer proposed Dempster's rule of combination where the conflict is reallocated proportionally among the other masses. Then Zadeh presented an example where Dempster's rule of combination produces unsatisfactory results. Several solutions were proposed: the TBM solution where masses are not renormalized and conflict is stored in the mass given to the empty set, Yager's solution where the conflict is transferred to the universe and Dubois and Prade's solution where the masses resulting from pairs of conflictual focal elements are transferred to the union of these subsets. Many other suggestions have then been made, creating a 'jungle' of combination rules. We discuss the nature of the combinations (conjunctive versus disjunctive, revision versus updating, static versus dynamic data fusion), argue about the need for a normalization, examine the possible origins of the conflicts, determine if a combination is justified and analyze many of the proposed solutions.
\end{abstract}

Keywords : Belief functions, transferable belief model, combination rules, conflict, belief normalization

\section{Introduction}

The transferable belief model (TBM) is a model for the quantified representation of epistemic uncertainty, i.e., the beliefs (or weighted opinions) held by a 'belief holder', called You hereafter and which can be a robot, an intelligent sensor, etc... Beliefs are represented by belief functions and, among others, by their related basic belief assignments (bba). Familiarity with belief function theory is assumed (see [98] for a recent updated survey).

Our paper concerns the models where belief functions are used to represent beliefs and that do not ask for an explicit underlying probability function. This covers the TBM [104] but also the model presented in Shafer's seminal book [79] which is essentially equal to the TBM except for the normalization phase we will study in this paper. Nevertheless many comments could be applied to the Dempsterian models, i.e., the model studied by Dempster [17, 18], those considered in Shafer's papers, the hint model [50], the probabilistic assumption model (PAS) [34]. These Dempsterian models ${ }^{1}$ are based on a meaningful ${ }^{2}$

\footnotetext{
${ }^{1}$ We do not use the term Dempster-Shafer's theory as it is confusing. Usually the term covers the Dempsterian models, but some authors use it for an upper and lower probability theory or a random set model or a probabilistic model extended to modal propositions.

${ }^{2}$ To be 'meaningful', a probability function must concern a variable on which either bets could be established and settled (for
} 
underlying probability function, i.e. probabilities which admit an operational definition like those based on betting ratios.

The TBM assumes that there is an object $o b$ and an attribute $X$ which depends on $o b$, whose actual value is denoted $\omega_{0}$ and is supposed to belong to a finite set of possible alternatives denoted $\Omega=\left\{\omega_{1}, \omega_{2}, \ldots, \omega_{n}\right\}$. The actual value $\omega_{0}$ is not known, only beliefs, weighted opinions, about its possible value are available. The operational definition of these beliefs is detailed in [100].

Assuming that $\omega_{0}$ is necessarily an element of $\Omega$ is called the closed world assumption. Assuming the possibility that $\omega_{0}$ might not be any of the alternatives listed in $\Omega$ is called the open world assumption. Its meaning is detailed in [87].

The belief about the value of $\omega_{0}$ is commonly produced by a given source and based on a piece of evidence collected by this source. Suppose the beliefs about $\omega_{0}$ are produced by two unrelated sources that use two distinct pieces of evidence. These two beliefs are then commonly combined by a conjunctive rule, the operator that performs the data fusion. Shafer proposes Dempster's rule of combination [79]. Then Zadeh presents an example where this rule produces results usually judged unsatisfactory [114, 115, 116]. One explanation was that the conflict was mismanaged by Dempster's rule of combination. Since Zadeh's paper, a jungle of alternative rules has bloomed, often leaving the user completely confused.

In fact most rules share the same basis. Suppose two bbas $m_{1}$ and $m_{2}$ defined on the frame of discernment $\Omega$ and let $m_{12}$ be the result of their combination. Let the function $f_{12}: 2^{\Omega} \rightarrow[0,1]$ with:

$$
f_{12}(X)=\sum_{A \cap B=X} m_{1}(A) m_{2}(B), \forall X \subseteq \Omega .
$$

We call it the conjunctive rule. Of course, the issue is to determine $m_{12}$. In the TBM, $m_{12}$ is equated to $f_{12}$, in which case $m_{12}(\emptyset)$ may be positive. For Dempster's rule of combination, $f_{12}(\emptyset)$ is proportionally reallocated and:

$$
m_{12}(X)=f_{12}(X)+f_{12}(\emptyset) \frac{f_{12}(X)}{1-f_{12}(\emptyset)}=\frac{f_{12}(X)}{1-f_{12}(\emptyset)} .
$$

Most other suggested rules are also based on $f_{12}$ and differ on the way they handle $f_{12}(\emptyset)$.

The purpose of this paper consists in detailing the assumptions that underlie the applicability of the combination rules. The correct understanding of these requirements can be used in order to build an expert system that verifies the applicability of the combination rules. Trying to fore a combination of two bbas that do not satisfy the applicability criteria is of course erroneous. We also survey many of the methods proposed to handle conflicts encountered when combining two belief functions, provided the applicability criteria are satisfied. We also study the essential properties they satisfy or not.

In [90], we already discuss the issue but new results justify their reconsideration. Recently [78] present a good survey of the combination rules. We add here some unconsidered rules. More importantly, we examine the assumptions required by the combination.

In this paper, we present the necessary background material in section 2 . In section 3 we discuss several issues related to the combination and the normalization of bbas. In section 4 , we present the difference between static and dynamic fusion and the consequence of this distinction on the choice of a combination rule. In section 5, we explain what are the assumptions that underlie the conjunctive combination rules. In section 6, we explain how we could handle the conflict. In section 7 , we conclude. In Appendix A, we summarize several technical points and list most combination rules discussed in this paper.

We realize that this paper may seem polemical at some places. It raises many issues for which we often present our own view, but we acknowledge that we are of course biased in favor of the TBM. Still we try to stay fair and skeptical. In any case, we did not want to hurt anybody and apologize to those who feel offended.

\section{Background material}

Some knowledge of the TBM and belief function theory is assumed. We list a few definitions to avoid misunderstanding (section 2.1) and fix the notation (section 2.2). We also discuss the concept of source reliability and its related discounting (section 2.3). Up to date details on the TBM can be found in [98].

the subjectivists) or frequencies of occurrence could be defined (for the frequentists). 


\section{$2.1 \quad$ Some definitions}

Definition 2.1 : Frame of discernment. The frame of discernment is a finite set of mutually exclusive elements, denoted $\Omega$ hereafter.

Beware that the frame of discernment is not necessarily exhaustive. Infinite frames of discernment [101] are not used in this paper.

Definition 2.2 : Basic belief assignment. A basic belief assignment (bba) is a mapping $\mathrm{m}^{\Omega}$ from $2^{\Omega} \rightarrow[0,1]$ that satisfies $\sum_{A \subseteq \Omega} m^{\Omega}(A)=1$. The basic belief mass $(b b m) m(A), A \subseteq \Omega$, is the value taken by the bba at $A$.

Definition 2.3 : Set of bbas. The set $\mathcal{B}^{\Omega}$ is the set of bbas defined on $\Omega$.

Definition 2.4 : Focal elements. The focal elements of a bba $m^{\Omega}$ are the subsets $A$ of $\Omega$ such that the bbm $m^{\Omega}(A)$ is positive.

Definition 2.5 : Categorical belief function. A categorical belief function on $\Omega$ focused on $A^{*} \subseteq \Omega$, is a belief function which related bba $\mathrm{m}^{\Omega}$ satisfies:

$$
m^{\Omega}(A)= \begin{cases}1 & \text { if } A=A^{*} \\ 0 & \text { otherwise. }\end{cases}
$$

When all bbas are categorical, the TBM becomes equivalent to classical propositional logic. Two limiting cases of categorical bbas have received special names.

Definition 2.6 : Vacuous belief function. The vacuous belief function on $\Omega$ is a categorical belief function focused on $\Omega$. It is denoted $V B F$.

Definition 2.7 : Contradictory belief function. A contradictory belief function on $\Omega$ is a categorical belief function focused on $\emptyset$.

Definition 2.8 : Normalized basic belief assignment. A normalized bba is a bba that satisfies $m(\emptyset)=$ 0 .

Definition 2.9 : Dogmatic basic belief assignment. A dogmatic bba on $\Omega$ is a bba that satisfies $m^{\Omega}(\Omega)=0$.

Definition 2.10 : Bayesian belief function. A Bayesian belief function on $\Omega$ is a belief function on $\Omega$ whose focal elements are singletons of $\Omega$.

A Bayesian belief function is just a probability function.

Definition 2.11 : Related functions. Given a bba $\mathrm{m}^{\Omega}$, one defines several related functions which are in one-to-one correspondence with $\mathrm{m}^{\Omega}$ :

$$
\begin{aligned}
\text { belieffunction } & \text { bel }(X) & =\sum_{A \subseteq X, A \neq \emptyset} m^{\Omega}(A) \\
\text { implicabilityfunction } & b(X) & =\sum_{A \subseteq X} m^{\Omega}(A) \\
\text { plausibilityfunction } & p l(X) & =\sum_{A \cap X \neq \emptyset} m^{\Omega}(A) \\
\text { commonalityfunction } & q(X) & =\sum_{X \subseteq A} m^{\Omega}(A)
\end{aligned}
$$

Definition 2.12 : The conjunctive rule. Given two bbas $m_{1}^{\Omega}$ and $m_{2}^{\Omega}$, the function $f_{12}$ that results from the application of the conjunctive rule is given by:

$$
f_{12}(A)=\sum_{X \cap Y=A} m_{1}^{\Omega}(X) m_{2}^{\Omega}(Y), \forall A \subseteq \Omega .
$$


Definition 2.13 : TBM conjunctive combination rule. Given two bbas $m_{1}^{\Omega}$ and $m_{2}^{\Omega}$, the bba that results from their conjunctive combination is given by $m_{1}^{\Omega} @ m_{2}^{\Omega}=m_{1}^{\Omega} @ 2$ where

$$
m_{1 @ 2}^{\Omega}=f_{12} \text {. }
$$

In the TBM literature, this rule is normally called the conjunctive combination rule. We call it here the TBM conjunctive combination rule in order to distinguish it from the other conjunctive combination rules we consider.

Definition 2.14 : Dempster's rule of combination. Given two bbas $m_{1}^{\Omega}$ and $m_{2}^{\Omega}$, the bba that results from the application of Dempster's rule of combination is given by $m_{1}^{\Omega} \oplus m_{2}^{\Omega}=m_{1 \oplus 2}^{\Omega}$ where:

$$
m_{1 \oplus 2}^{\Omega}(A)= \begin{cases}\frac{f_{12}(A)}{1-f_{12}(\emptyset)}, & \forall A \subseteq \Omega, A \neq \emptyset \\ 0 & \text { if } A=\emptyset\end{cases}
$$

We give the definition of two other combination rules. In Yager's combination rule [112, 113, 49], the mass $f_{12}(\emptyset)$ is transferred to $\Omega$. In Dubois and Prade's combination rule [23], whenever two focal elements have an empty intersection, the product of their masses is allocated to the union of the two focal elements.

Definition 2.15 : Yager's combination rule. Given two bbas $m_{1}^{\Omega}$ and $m_{2}^{\Omega}$, the bba $m_{12}$ that results from the application of Yager's combination rule is given by:

$$
m_{12}^{\Omega}(A)= \begin{cases}f_{12}(A) & \forall A \subseteq \Omega, A \neq \Omega, A \neq \emptyset \\ f_{12}(\Omega)+f_{12}(\emptyset) & \text { if } A=\Omega \\ 0 & \text { if } A=\emptyset\end{cases}
$$

Definition 2.16 : Dubois and Prade's combination rule. Given two bbas $m_{1}^{\Omega}$ and $m_{2}^{\Omega}$, the bba $m_{12}$ that results from the application of Dubois and Prade's combination rule is given by:

$$
m_{12}^{\Omega}(A)= \begin{cases}f_{12}(A)+\sum_{\substack{A \cap B=\emptyset, A \cup B=X}} m_{1}(A) m_{2}(B) & \forall A \subseteq \Omega, A \neq \emptyset \\ 0 & \text { if } A=\emptyset\end{cases}
$$

In the TBM, decisions are made using the pignistic probabilities derived from the bba by the pignistic transformation.

Definition 2.17 : The pignistic transformation. The pignistic transformation maps bbas to so called pignistic probability functions. The pignistic transformation of $m^{\Omega}$ is given by:

$$
\operatorname{Bet}^{\Omega}(A)=\sum_{B \subseteq \Omega} \frac{|A \cap B|}{|B|} \frac{m^{\Omega}(B)}{1-m^{\Omega}(\emptyset)}, \forall A \subseteq \Omega
$$

where $|A|$ is the number of elements of $\Omega$ in $A$.

\subsection{Notation}

We use the next notation to express the beliefs, or its related bba $m$, held by You at a given time $t$ about the actual value $\omega_{0}$ of an attribute of an object $o b$ which possible values belong to the frame of discernment $\Omega$, a finite set, all this based on the evidential corpus $E C_{\text {You, } t}$ held by You at $t$ :

- for the belief function: $b e l_{\mathrm{You}, t}^{\Omega}\{o b\}\left[E C_{\mathrm{You}, t}\right]$

- for the bba: $m_{\text {You }, t}^{\Omega}\{o b\}\left[E C_{\text {You }, t}\right]$

both mapping $2^{\Omega}$ to $[0,1]$ and where $E C_{\text {You, } t}$ is a set of propositions accepted as true by You at $t$ when constructing Your beliefs.

Many indexes are neglected when the context makes them obvious. 


\subsection{Source reliability and discounting beliefs}

Suppose a source $S$ is producing a bba $m_{S}^{\Omega}$ representing the source beliefs about the actual value $\omega_{0}$ of a variable which domain is $\Omega$. This bba can be any bba in $\mathcal{B}^{\Omega}$. S is communicating this bba to You. Suppose furthermore that You had no idea about what might be $\omega_{0}$, i.e., suppose Your prior belief on $\omega_{0}$ is a vacuous belief function.

Consider the case where You accept that $S$ is fully reliable, i.e., You believe what $S$ states. In that case Your belief about $\omega_{0}$ is the bba produced by $S$.

Consider the case where You accept that $S$ is not reliable, i.e., You do not believe what $S$ states. In that case Your belief about $\omega_{0}$ is the vacuous belief function.

Consider now the intermediate case where You accept that $S$ might be more or less unreliable, not trustworthy, i.e., You may believe more or less what $S$ states. If the source was reliable, You would believe what it states. If the source was not reliable, You would discard what it states. More formally, it means You would build a bba on the space $\{R, N R\}$ representing Your beliefs about the fact that $S$ is reliable $(R)$ or is not reliable $(N R)$. If You accept that $S$ is reliable then Your belief about $\omega_{0}$ would be $S$ 's beliefs. If You accept that $S$ is not reliable then Your belief about $\omega_{0}$ would be a state of total ignorance, thus the vacuous belief function.

Let $\alpha=m_{\text {You }}^{\{R, N R\}}(R)$ be Your belief that $S$ is reliable. Then the application of the General Bayesian Theorem $[86,92,16]$ produces Your belief on $\Omega$.

$$
m_{\text {You }}^{\Omega}\left[\alpha, m_{S}^{\Omega}\right](X)= \begin{cases}\alpha m_{S}^{\Omega}(X) & \text { if } X \neq \Omega \\ \alpha m_{S}^{\Omega}(\Omega)+(1-\alpha) & \text { if } X=\Omega .\end{cases}
$$

This transformation of a bba is called a discounting [79]. Its justification as just described is presented in [92]. Every mass is shrunk by a factor $\alpha$ and the mass lost during this process is transferred to the universe. $\alpha$ is called the coefficient of reliability and $\beta=1-\alpha$ is called the discount rate. The notations $m^{\alpha}$ and $m^{\Omega, \alpha}$ are used to denote the bbas that result from the discounting of the bbas $m$ and $m^{\Omega}$, respectively. Note the exponent coefficient is the reliability factor; this choice simplifies later notations.

We present two properties of the discounting. Discounting the vacuous belief function produces the vacuous belief function.

Lemma 2.1 $V B F^{\alpha}=V B F$.

Iterated discounting is still a discounting and the final reliability coefficient is the product of the individual reliability coefficients.

Lemma 2.2 Let the bba $m$ and $\alpha_{1}, \alpha_{2} \in[0,1]$. Then:

$$
\left(m^{\alpha_{1}}\right)^{\alpha_{2}}=m^{\alpha_{1} \alpha_{2}} .
$$

Proof. We have: $m^{\alpha_{1}}(X)=\alpha_{1} m(X)$ for $X \neq \Omega$ and $m^{\alpha_{1}}(\Omega)=\left(1-\alpha_{1}\right)+\alpha_{1} m(\Omega)$. We then discount $m^{\alpha_{1}}$ at rate $1-\alpha_{2}$. The result is given by $\alpha_{2} \alpha_{1} m(X)$ for $X \neq \Omega$ and $\left(1-\alpha_{2}\right)+\alpha_{2}\left(1-\alpha_{1}\right)+\alpha_{2} \alpha_{1} m(\Omega)$ which is equal to $\left(1-\alpha_{2} \alpha_{1}\right)+\alpha_{2} \alpha_{1} m(\Omega)$.

\section{Combining beliefs}

Suppose two 'distinct' pieces of evidence $E v_{1}$ and $E v_{2}$ and let their induced bbas $m^{\Omega}\left[E v_{i}\right]$ be denoted by $m_{i}$ for $i=1,2$. These two bbas must be combined in order to perform data fusion. Several issues about such a combination are examined so the reader can see what they are.

We first define distinctness (section 3.1) a concept which is fundamental for the applicability of all the combination rules analyzed here and too often taken for granted by users, while it must be explicitly justified. We then emphasize that combinations are not always conjunctive. Other forms exist among which the disjunctive rule of combination plays a role in some rules (section 3.2). We detail some of the justifications that have been proposed for some of the conjunctive combination rules (section 3.3). We present a short history of the origin of the 'jungle' of combination rules developed in the literature essentially to answer to Zadeh's counterexample (section 3.4). We argue about the need for normalization (section 3.5) and its relation with decision making (section 3.6). We show how source and report ratings are handled (section 3.7) before concluding this section (section 3.8). 


\subsection{Defining distinctness}

A first understanding of distinctness can result from the examination of what Dempster's rule of combination becomes when the bbas are probability functions. It turns out that in this degenerated case, distinctness becomes essentially equal to conditional independence with an equi a priori probability.

Theorem 3.1 Let $m_{1}^{\Omega}$ and $m_{2}^{\Omega}$ be the bbas related to two Bayesian belief functions induced by two pieces of evidence $E v_{1}$ and $E v_{2}$, respectively. For $i=1,2$, define $p\left(\omega \mid E v_{i}\right)=m_{i}^{\Omega}(\omega)$ for all $\omega \in \Omega$. If $E v_{1}$ and $E v_{2}$ are conditionally stochastically independent given every $\omega \in \Omega$, and if the a priori probability function on $\Omega$ is given by $p(\omega)=1 /|\omega|$ for all $\omega \in \Omega$, then $p\left(. \mid E v_{1}, E v_{2}\right)=m_{1}^{\Omega} \oplus m_{2}^{\Omega}$ where $\oplus$ denotes Dempster's rule of combination.

Proof. One has:

$$
\begin{aligned}
& p\left(\omega \mid E v_{1}, E v_{2}\right)= \\
& =k \cdot p\left(E v_{1}, E v_{2} \mid \omega\right) \cdot p(\omega) \quad \text { Bayes theorem } \\
& =k \cdot p\left(E v_{1} \mid \omega\right) \cdot p\left(E v_{2} \mid \omega\right) \cdot p(\omega) \quad \text { conditional independence } \\
& =k \cdot p\left(\omega \mid E v_{1}\right) \cdot p\left(\omega \mid E v_{2}\right) \cdot p\left(E v_{1}\right) \cdot p\left(E v_{2}\right) / p(\omega) \quad \text { twice Bayes theorem }
\end{aligned}
$$

where

$$
k=\sum_{\omega_{i} \in \Omega} p\left(\omega_{i} \mid E v_{1}\right) \cdot p\left(\omega_{i} \mid E v_{2}\right) \cdot p\left(E v_{1}\right) \cdot p\left(E v_{2}\right) / p\left(\omega_{i}\right) .
$$

As $p(\omega)$ is constant, we get:

$$
p\left(\omega \mid E v_{1}, E v_{2}\right)=\frac{p\left(\omega \mid E v_{1}\right) \cdot p\left(\omega \mid E v_{2}\right)}{\sum_{\omega_{i} \in \Omega} p\left(\omega_{i} \mid E v_{1}\right) \cdot p\left(\omega_{i} \mid E v_{2}\right)} .
$$

This relation can be written as:

$$
m^{\Omega}\left[E v_{1}, E v_{2}\right](\omega)=\frac{m^{\Omega}\left[E v_{1}\right](\omega) \cdot m^{\Omega}\left[E v_{2}\right](\omega)}{\sum_{\omega_{i} \in \Omega} m^{\Omega}\left[E v_{1}\right]\left(\omega_{i}\right) \cdot m^{\Omega}\left[E v_{2}\right]\left(\omega_{i}\right)}
$$

thus the result obtained by Dempster's rule of combination.

This property cannot be extended to general belief functions. For these belief functions, the concept of distinctness (also called 'independence', maybe a unfortunate expression) was not formalized (nevertheless see $[82,57,98$, page 279]. Recently $[5,6]$ the concept of 'doxastic independence' was defined. Its relation to distinctness is detailed in Appendix A.1.

In practice, distinctness can be defined as follows. Suppose two variables $X$ and $Y$. Let the bbas $m^{X}$ and $m^{Y}$ represent the beliefs relative to the actual value of the variables. Suppose the two bbas are induced by two pieces of evidence.

Definition 3.1 : Distinctness. The two pieces of evidence that induce $m^{X}$ and $m^{Y}$ are distinct iff the bba $m^{X \times Y}$ can be computed from $m^{X}$ and $m^{Y}$ by applying the product rule, i.e., where $\forall A \subseteq X \times Y$ :

$$
m^{X} @ m^{Y}(A)= \begin{cases}m^{X}(B) m^{Y}(C) & \text { if } A=B \times C, B \subseteq X, C \subseteq Y, \\ 0 & \text { otherwise }\end{cases}
$$

(see details in section 5.1).

This definition is the analogous of the definition $P(A \cap B)=P(A) P(B), \forall A, B$ as used for stochastic independence in probability theory. If furthermore the variables $X$ and $Y$ refer to the same variable, we add this new information and the result is the TBM conjunctive combination rule (see relation (4)). 


\subsection{Types of combinations}

Given two distinct pieces of evidence $E v_{i}$ 's and their induced $m\left[E v_{i}\right], i=1,2$, You can build several combinations.

- $m\left[E v_{1} \wedge E v_{2}\right]$ : the belief that results from the conjunctive combination of the two pieces of evidence. It applies when You accept that the two $E v_{i}$ 's hold.

- $m\left[E v_{1} \vee E v_{2}\right]$ : the belief that results from the disjunctive combination of the two pieces of evidence. It applies when You accept that at least one of the two $E v_{i}$ 's holds but You do not know which one.

- $m\left[E v_{1} \underline{\vee} E v_{2}\right]$ : the belief that results from the exclusive disjunction combination of the two pieces of evidence. It applies when You accept that one and only one of the two $E v_{i}$ 's holds but You do not know which one.

To fix the idea, we list here the solutions proposed within the TBM for these three combinations. For all $A \subseteq \Omega$ :

conjunctive combination rule

$$
m\left[E v_{1} \wedge E v_{2}\right](A)=\sum_{B, C \subseteq \Omega, B \cap C=A} m\left[E v_{1}\right](B) m\left[E v_{2}\right](C)
$$

disjunctive combination rule

$$
m\left[E v_{1} \vee E v_{2}\right](A)=\sum_{B, C \subseteq \Omega, B \cup C=A} m\left[E v_{1}\right](B) m\left[E v_{2}\right](C)
$$

exclusive disjunction combination

$$
m\left[E v_{1} \underline{\vee} E v_{2}\right](A)=\sum_{B, C \subseteq \Omega, B \underline{\cup} C=A} m\left[E v_{1}\right](B) m\left[E v_{2}\right](C)
$$

where $B \underline{\cup} C=(B \cap \bar{C}) \cup(\bar{B} \cap C)$.

In fact any set of logical connectives can be considered thanks to the concept of the negation of a belief function [23].

All these combination rules belong to the general mathematical family of the so called $\alpha$-junctive combination rules [95]. In the present paper, we will essentially use the conjunctive rules.

\subsection{Axiomatic justification of the combination rules}

The interest of an axiomatic justification of a rule comes from the fact the axioms, normally, are properties that can be analyzed individually and judged as acceptable and/or required. Just listing axioms is hardly convincing. Their meanings must be evaluated. In particular, the user must consider what would happen if an axiom was not satisfied. Axioms have been proposed for Dempster's rule of combination within the Dempsterian models that assumes a meaningful underlying probability function, and for the TBM conjunctive combination rule within the TBM. We are not aware of similar axiomatic justifications for the other rules.

\subsubsection{Justification of Dempster's rule of combination for the Dempsterian models}

In the Dempsterian models, Dempster's rule of combination results from a direct application of probability theory and of the concept of stochastic independence. Detailed justification can be found in [82] (see also [21, pages 125-129]).

Let $X$ and $Y$ be two stochastically independent random variables, with $P^{X}$ and $P^{Y}$ their probability measures, respectively. Let a space $\Omega$ and the one-to-many mappings $\Gamma_{X}: X \rightarrow 2^{\Omega}$ and $\Gamma_{Y}: Y \rightarrow 2^{\Omega}$. Suppose the next two sets of rules and their consequence where $\omega_{0}$ denotes the actual value of $\Omega$ : 
For all $x \in X$, if $X=x$, then $\omega_{0} \in \Gamma_{X}(x)$

and the probability of the premise is $P^{X}(x)$.

For all $y \in Y$, if $Y=y$, then $\omega_{0} \in \Gamma_{Y}(y)$

and the probability of the premise is $P^{Y}(y)$.

Thus

For all $x \in X$ and $y \in Y$, if $X=x$ and $Y=y$, then $\omega_{0} \in \Gamma_{X}(x) \cap \Gamma_{Y}(y)$

and the probability of the premise is $P^{X}(x) P^{Y}(y)$.

From $P^{X}$ and $\Gamma_{X}$, we define the bba:

$$
m_{X}^{\Omega}(A)=P^{X}\left(\left\{x: \Gamma_{X}(x)=A\right\}\right)=\sum_{x \in X: \Gamma_{X}(x)=A} P^{X}(x), \forall A \subseteq \Omega .
$$

We do similarly for $m_{Y}^{\Omega}(A)$. Then the bba based on both sets of rules is given by

$$
m_{X, Y}^{\Omega}(A)=\sum_{x, y: \Gamma(x) \cap \Gamma_{y}(y)=A} P^{X}(x) P^{Y}(y) .
$$

It can be shown that $m_{X, Y}^{\Omega}$ is the bba one obtains by combining $m_{X}^{\Omega}$ and $m_{Y}^{\Omega}$ by the conjunctive combination (3).

If we add the constraints $\Gamma_{X}(x) \neq \emptyset, \forall x \in X$ and $\Gamma_{Y}(y) \neq \emptyset, \forall y \in Y$ and we condition $m_{X, Y}^{\Omega}$ on $\Gamma(x) \cap \Gamma_{y}(y) \neq \emptyset$ in relation (14), the result is Dempster's rule of combination, i.e., the same as (14) except all terms are divided by $1-m_{X, Y}^{\Omega}(\emptyset)$ and then we put $m_{X, Y}^{\Omega}(\emptyset)=0$.

As can be seen, the normalization requirement, even though quite natural, is not necessary. For instance, why do we require that $\Gamma_{X}(x) \neq \emptyset$ ? One can imagine that some $x \in X$ maps on the empty set of $\Omega \in 2^{\Omega}$. In fact, at the risk of being an iconoclast, we can wonder if the property $P(\Omega)=1$ assumed among the Kolmogoroff axioms was really necessary.

\subsubsection{Justification of conjunctive combination rule in the TBM}

To justify the conjunctive combination rule within the TBM framework, we may not use the probabilities used for the Dempsterian cases as done in the previous section.

In the TBM, the justification of the TBM conjunctive combination rule is based on associativity and commutativity. It was first axiomatically justified in [88, 47, 35]. Still [48] presents a better justification based on the concept of specialization introduced in $[109,51]$. The concept of specialization and its relation with the conjunctive combination rule are detailed in [98].

Remember that, in the TBM, $m(A)$ is the part of belief that supports that the actual value $\omega_{0}$ belongs to $A \subseteq \Omega$ and nothing more specific because of a lack of knowledge. Suppose You receive a new piece of information relative to $\omega_{0}$. Your beliefs must then be revised. The conjunctive nature of the considered combination means that knowledge increases monotonically, i.e., new knowledge does not lead to the necessity of retracting some of the previous knowledge ${ }^{3}$. You knew that $m(A)$ was supporting $\omega_{0} \in A$ and nothing more specific because of the lack of information. Now You get some new information, so $m(A)$ must be reallocated to more specific subsets of $A$ ('masses flow down'). This transfer of masses is called a specialization. Moving (part of) $m(A)$ to sets $B$ which are not subsets of $A$ would violate the monotonicity.

Definition 3.2 : Specialization. A specialization $S^{\Omega}$ is a mapping from $\mathcal{B}^{\Omega}$ to $\mathcal{B}^{\Omega}$ which coefficients $s(A, B)$ for $A, B \subseteq \Omega$ satisfy: $s(A, B) \geq 0, s(A, B)=0$ if $A \nsubseteq B$ and $\sum_{A} s(A, B)=1$. The bba $m_{1}^{\Omega}$ resulting from the application of the specialization operator on the bba $m_{0}^{\Omega}$ satisfies:

$$
m_{1}^{\Omega}(A)=\sum_{B \subseteq \Omega} s(A, B) m_{0}^{\Omega}(B)
$$

The bba $m_{1}^{\Omega}$ is called a specialization of $m_{0}^{\Omega}$. The bba $m_{1}^{\Omega}$ is said to be more committed than $m_{0}^{\Omega}$.

\footnotetext{
${ }^{3}$ The TBM has been extended to non-monotonic reasoning and default reasoning in [7]
} 
The term $s(A, B)$ represents the 'part' of the bbm $m_{0}^{\Omega}(B)$ transferred to $A$ by $S^{\Omega}$.

The conditioning rule and the TBM conjunctive combination rule are derived from the specialization principle as proven in [48].

We first derive the conditioning rule. We require that:

1. conditioning is represented by a specialization. This reflects the monotonicity of the conjunctive nature of the conditioning process.

2. after conditioning a bba $m^{\Omega}$ on $A \subseteq \Omega$, the complement $\bar{A}$ should not be plausible anymore: $p l_{1}^{\Omega}[A](\bar{A})=$ 0 . Once You accept as true that $\omega_{0} \in A$, how could You still consider that $\bar{A}$ is still plausible.

3. among all possible solutions satisfying the previous two requirements, we select the least committed one, i.e., the bba such that all other possible solutions are more committed than itself. This reflects the principle that one should never allocate more belief than justified [36].

The only solution is given by the unnormalized Dempster's rule of conditioning.

Theorem 3.2 Given a bba $m_{0}^{\Omega}$ and $A \subseteq \Omega$, let $S\left(m_{0}^{\Omega}, A\right)$ be the set of all its specializations $m_{1}^{\Omega}$ of $m_{0}^{\Omega}$ such that $p l_{1}^{\Omega}(\bar{A})=0$. The least committed element of $S\left(m_{0}^{\Omega}, A\right)$ satisfies:

$$
m_{1}^{\Omega}(B)=m_{0}^{\Omega}[A](B)= \begin{cases}\sum_{C \subseteq \bar{A}} m_{0}^{\Omega}(B \cup C), & \text { if } B \subseteq A \\ 0 & \text { otherwise }\end{cases}
$$

The only associative and commutative specializations which generalize this conditioning rule correspond to the TBM conjunctive combination rule.

Theorem 3.3 Suppose three bbas $m_{i}^{\Omega}, m_{j}^{\Omega}$ and $m_{k}^{\Omega}$. Assume their combinations must satisfy the following requirements.

1. $m_{i j}^{\Omega}$ is a specialization of $m_{i}^{\Omega}$ and $m_{j}^{\Omega}$,

2. $m_{i j}^{\Omega}=m_{j i}^{\Omega}$ (commutativity),

3. $m_{(i j) k}^{\Omega}=m_{i(j k)}^{\Omega}$ (associativity),

4. $m_{i A}^{\Omega}=m_{i}^{\Omega}[A]$ where $m_{A}^{\Omega}$ is a categorical bba focused on $A \subseteq \Omega$ and $m_{i}^{\Omega}[A]$ is given by (2).

Then $m_{i j}^{\Omega}=m_{i}^{\Omega} @ m_{j}^{\Omega}$.

In practice this theorem means that the only combination that results from an associative and commutative specialization is the TBM conjunctive combination rule. The axiomatic justification of the conditioning and combination rules is thus based on the concept of specialization, associativity, commutativity, least commitment and the requirement $p l^{\Omega}[A](\bar{A})=0$ ? Each of these requirements seem natural, and to reject the TBM conjunctive combination rule, one must show and justify which of these requirements is rejected. In particular, normalizing the result would violate the specialization requirement.

\subsubsection{Dubois and Prade's justification}

In [22], Dubois and Prade prove that the product is the unique solution for $\otimes$ if one asks that the combination satisfies the next algebraic property:

$$
m_{12}^{\Omega}(X)=\sum_{A \cap B=X} m_{1}^{\Omega}(A) \otimes m_{2}^{\Omega}(B), \forall X \subseteq \Omega
$$

where $m_{1}^{\Omega}$ and $m_{2}^{\Omega}$ are given bbas and $m_{12}^{\Omega}$ is the bba that would result by their combination based on a continuous 2-place operator $\otimes$. This also justifies the TBM conjunctive combination rule. 


\subsubsection{Other combination rules}

The other combinations rules examined in this paper are usually not based on such kinds of justification. They were created to handle what their authors consider as an unsatisfactory property, the non null mass given to $\emptyset$. Usually these rules are shown to have a few good behaviors for particular combinations. Even though these rules are often quite natural, they are usually ad hoc in that they lack any real justification.

Just to emphasize the issue, we could imagine a combination rule where the masses are randomly allocated among the subsets of the frame of discernment. Common sense dictates that such a rule is inadequate. When creating new rules, authors should have developed criteria that are satisfied by their rules and that at least reject this kind of rule. Unfortunately, such criteria are often missing. The pragmatic fact 'Our rule works fine' is of course not a real justification, at most a necessary condition.

Nevertheless, being ad hoc is not necessarily as bad as it seems. The advantage of a well founded rule comes from the opinion that knowing the properties of a rule would protect its user from disappointment. Still some ad hoc rules might behave better, and there are many such examples in real life. So being ad hoc is theoretically bad, but we must abstain from any definitive judgment.

\subsection{The jungle of combination rules}

Shafer [79] considers that beliefs are defined on a frame of discernment $\Omega$, i.e. a set of mutually exclusive and exhaustive alternatives, as Shafer accepts the closed world assumption. Shafer considers only the conjunctive combination and proposes Dempster's rule of combination, a rule that was already implicitly described in $[17]$.

Then Zadeh presented his by now famous counterexample $[114,116]$, and many solutions were subsequently proposed to solve it.

Zadeh's counterexample. Let the two bbas $m_{1}$ and $m_{2}$ defined on $\Omega=\{a, b, c\}$. Let

$$
\begin{array}{lll}
m_{1}(a)=0.99, & m_{1}(b)=0.01 \\
m_{2}(c)=0.99, & m_{2}(b)=0.01
\end{array}
$$

The application of Dempster's rule of combination on these two highly conflicting bbas produces a bba $m$ with $m(b)=1$. So the $b$ alternative, hardly supported by each piece of evidence, turns out to be fully supported aftre the combination of the two bbas.

Shafer argues that this should be the solution as there are only three alternatives, one must hold, and thus only $b$ resists. This results from a strict application of the closed world assumption and the Dempsterian nature of his model ${ }^{4}$.

Very quickly, other answers were proposed that all reject the $m(b)=1$ solution. Yager (equation 6 ) defends that the conclusion should be $m(b)=0.0001, m(\Omega)=.9999$, which enhances the user's state of confusion, thus of ignorance, induced by the two highly conflicting pieces of evidence [112, 113]. Simultaneously, we (equation 4) suggest to leave the 0.9999 conflict on the empty set, thus violating Shafer's requirement $m(\emptyset)=0$. It was the starting point in the creation of the TBM [104]. Dubois and Prade (equation 7) suggest a solution more flexible than Yager's solution for the transfer of the conflictual masses [23]. Their solution kept the $m(\emptyset)=0$ assumption and is somehow in the spirit of Yager's solution.

Then alternative solutions start blooming, most feeling, rightly or wrongly, that the TBM solution, i.e., allocating conflict to the empty set, is hard to accept. Others kept defending Dempster's rule of combination [32] or showing that Zadeh's counterexample is not a counterexample [33]. Surprisingly, hardly anybody seems to have realized that this example can also be used against probability theory ${ }^{5}$

Meanwhile another counterexample shows up.

\footnotetext{
${ }^{4}$ It satisfies Sherlock Holmes' famous statement: 'When you have eliminated the impossible, whatever remains, however improbable, must be the truth.'

${ }^{5}$ Thanks to the referee who points it to me.
} 
The bomb counterexample. Suppose two sensors observing an object air-launched, one reports the object is a large bomb, the second that it is a small bomb. For a bomb, being large and small is assumed to be a contradiction. Combining these two facts (represented by categorical bbas) by the TBM conjunctive combination rule ends up with another categorical bba which allocates a mass 1 to the contradiction 'large and small and bomb'. Several users defend that the conclusion 'it is a bomb' should be supported (as it happens to be the case if we apply Dubois and Prade's combination rule).

Some authors $[8,39,67]$ propose to replaced the classical Boolean algebras by a distributive lattice [44]. Their proposed extension unfortunately did not solve the bomb issue (see Appendix A.2).

More recently [20, 84] develop their so called Dezert-Smarandache Theory (DSmT) of plausible and paradoxical reasoning where they also replace the Boolean algebras by a distributive lattice (these authors called it the hyper-power set). One of their motivations was to resolve Zadeh's counterexample and to provide some 'better' solutions for examples with large conflicts. For the bomb counterexample, their solution is identical to Dubois and Prade's solution.

\subsection{Normalizing or not?}

Normalizing a bba means requiring that $m(\emptyset)=0$ and that the sum of all bbms given to the non empty subsets is 1. In Dempster-Shafer's theory (whatever means that expression [94]) bbas are normalized. In the TBM, bbas are not normalized.

In fact, the TBM is apparently the only model where $m(\emptyset)>0$ is allowed and kept as such. All other models start by applying a combination rule that is initially often analogous to the one described in the TBM conjunctive combination rule, but followed by a form of normalization achieved by redistributing the mass allocated to the empty set among the other subsets of the frame of discernment, so at the end of the combination process, $m(\emptyset)=0$ is recovered. The reason for such a normalization often results from what we feel is a misunderstanding of the meaning of $m(\emptyset)$.

The justification for normalizing depends on the model underlying the use of belief functions, a distinction usually overlooked in the literature.

The Dempsterian model case In the Dempsterian models, one claims the existence of a space $X$ and of a probability function on $X$. Then one assumes the existence of a space $Y$ and of a one-to-many mapping $\Gamma: X \rightarrow 2^{Y}$ from the space $X$ to the space $Y$. The 'measure' induced on $Y$ by the probability function defined on $X$ and the mapping $\Gamma$ is not a probability measure, but a belief function. Suppose $x \in X$ is mapped on the empty set, which is the equivalent of the contradiction in classical logic. If such an $x$ prevails, than a 'logical contradiction' holds, what is not acceptable in classical logic. So the probability of such $x$ 's must be null. Hence the necessity to condition the probability function defined on $X$ on $\Gamma(x) \neq \emptyset$, which results in the normalization of the bbas defined on $Y$. So normalization is well justified in the Dempsterian models.

The TBM case In the TBM, the scenario is different. The primitive is the bba itself. It is essential to understand that the TBM is a non-probabilistic model, developed independently of the existence of any underlying probability function, The mass $m(A), A \subseteq \Omega$, is that part of Your belief supporting that the actual world belongs to $A$ and nothing more specific. No underlying $X$ space endowed with a meaningful probability function is assumed as would be the case with the Dempsterian models and the random set approach $[65,91,66,59,58,27]$. It is of course true that in the TBM, one can always define a space $X$, an probability function on $X$ and a $\Gamma$ mapping so that the TBM can be mathematically assimilated to a Dempsterian model, but could one bet on the actual value of $X$ or built frequencies on the values of $X$. The TBM covers both cases, where meaningful probability function are defined and where they are not. Hence the TBM covers the Dempsterian models as a special case.

In the case the TBM is not a Dempsterian model, nothing forbids that a non null mass is given to the empty set. We must only explain what $m(\emptyset)$ represents. In the TBM, $m(\emptyset)$ has at least three origins.

- The frame of discernment $\Omega$ is not exhaustive, i.e., some alternatives have been forgotten when establishing $\Omega[87]$ 
- There is a conflict between the bbas reported by two reliable sources of beliefs.

- There is a positive belief that the two sources of bbas do not report on the same object $[70,71,74,9,76]$ (see details in sections 5.2 and 6.2.3).

The fact that these various origins for $m(\emptyset)$ cannot be distinguished is felt by some authors as a weakness of the TBM. The extension of belief functions on non Boolean algebras tries somehow to answer to this criticism as the masses given to the various contradictory subsets are kept differentiated and not pooled into one mass given to $\emptyset$ as done in the TBM $[8,84,15]$.

Closing the world Another suggestion has been advocated for resolving the open world case [110, 69, 31]. It is based on adding an extra alternative $\omega^{*}$ to $\Omega$ that covers all those possible alternatives not initially mentioned is $\Omega$. Let $\Omega^{*}=\Omega \cup\left\{\omega^{*}\right\}$ denote this extended frame. This alternative $\omega^{*}$ is usually advocated in probability theory. In belief function theory, this solution nevertheless raises a problem. You must assess all the masses on $\Omega^{*}$ based on those assessed on $\Omega$. So You must decide for every $A \subseteq \Omega$ which part is to be allocated to $A$ and which to $A \cup\left\{\omega^{*}\right\}$. As far as You know nothing about $\omega^{*}$, it is hard to justify these repartitions. All You can easily defend is that You apply the least committed solution, in which case the whole mass initially given to $A$ is reallocated to $A \cup\left\{\omega^{*}\right\}$. In that case conflict disappears completely as $\omega^{*}$ belongs to every masses of every bba defined on $\Omega^{*}$. Such a solution must be handled with care when decision is involved as You need the cardinality of $\omega^{*}$. Furthermore what would You do if $\omega^{*}$ turns out to be the most probable solution. We suggest that before computing the pignistic probabilities, You should condition the bba on $\Omega$ - in which case You recover exactly the same bba as the one You would have computed within the TBM - and then proceed with the pignistic transformation.

\subsection{Normalization and decision making}

When developing a model for the belief representation, it is also necessary to develop a model for decision making, thus to explain how decisions can be made in an uncertain environment. In the Dempsterian models, some solutions are based on relative plausibilities or weighted expected utilities [2, 100, 11]. In the TBM, the solution is the pignistic transformation which is described in $[89,104,100]$ and justified in [102]. Some generalizations of the pignistic transformation are proposed in [106]. Other generalized pignistic transformation has been also analyzed in the distributive lattice context [84].

The issue of normalization seems often related to decision making, as a mass on the empty set might be a source of difficulty. In fact in the TBM, the pignistic transformation includes a normalization phase as every mass is divided by $(1-m(\emptyset))$. A casual reader may wonder if this is not just a hidden way to transform the TBM conjunctive combination rule into the Dempster's rule of combination. The difference is due to the fact that this concerns the pignistic level, where decisions must be taken, not the credal level where beliefs are held and manipulated [104]. At the credal level beliefs are not normalized, whereas at the pignistic level the pignistic probabilities must be normalized. The lack of normalization for the bbas at the credal level is not an issue for decision making.

Just to illustrate this reasoning, consider Zadeh's counterexample. The TBM allocates the masses $m(b)=$ $.0001, m(\emptyset)=.9999$ which indicates the existence of an enormous conflict. The pignistic transformation produces $\operatorname{Bet} P(b)=1$. Indeed if You must decide among the limited set of alternatives $\{a, b, c\}$, the only supported one is $b$. In fact Shafer's reply to Zadeh (nicely illustrated by Sherlock Holmes' citation) concerns more the pignistic probabilities than the bba. For decision making, open world cannot be considered as such, all alternatives must be defined before making decision.

The only case where the pignistic transformation cannot be computed is when full inconstancy prevails, i.e., when $m(\emptyset)=1$. This means that the sources of beliefs contradict each other completely. There is no hope for solving such an issue. The see why, consider the logical case where You know that $p$ and $\neg p$ both hold, and Your decision depends on $p$. There is no way to resolve this complete conflictual situation, and this is what the TBM will conclude. Note Dempster's rule of combination cannot be applied in such a case. On the other side, Yager's rule, Dubois and Prade's rule and many of those that normalize would put a mass 1 on the universe, and transform the full inconsistency into full ignorance, what is maybe arguable. Imagine three bbas, a categorical bba focused on $A$, another categorical bba focused on $\bar{A}$, the third being any bba $m^{*}$. Combine then conjunctively into $m$. In the TBM, the conflict persists and the result is $m(\emptyset)=1$ 
whatever $m^{*}$, indicating the presence of a conflict. With the other mentioned rules, the result is $m^{*}$ as if the conflict did not exist, what we feel unsatisfactory. This criteria is among those considered in section A.4.

\subsection{Rating sources and reports}

In intelligence gathering, one must rate separately the quality (reliability, degree of trustworthiness) of both the source that produces the report and the content of the report itself. In practice, if the source is judged 'unreliable', the report is essentially discarded: this is exactly what discounting performs. If the source is judged 'reliable', then the content of the report is evaluated to decide how much trust should be given to it. The mass given to the empty set is a measure of this trust. If the conflict is small, it means the report fits with previous opinions, and seems thus to reinforce them. If the conflict is large, it means that the content of the report clashes with the previous opinions. User must then find out the origin of the clash, and try to resolve it. For instance, the report may concern another object. Resolving the conflict has to be done by some external expert system built on top of the belief function system itself and that deals with the value to be attached to the various components of the report. This system is of course totally domain dependent.

\subsection{Concluding remarks on normalization}

My questions, as a biased defender of the TBM, are: Why to normalize at the credal level? Why to worry about the mass given to the empty set? Why to try to redistribute it in order to end up with $m(\emptyset)=0$ ? In the TBM, we just keep the conflictual mass on the empty set as an alarm signal for the existence of a conflict between the bbas, signal that triggers an expert system whose role is to find out the origin of the conflict (see section 6.1). No normalization is really required at the credal level.

\section{Static and dynamic fusion}

Data fusion (or combination) can be seen as a static or as a dynamic problem. In static fusion, all belief functions are combined simultaneously. In dynamic fusion, a sequence of belief functions are collected sequentially one by one. This fusion process results in a revision or an update of the current belief function. The distinction between belief revision and belief updating is detailed in the next section.

\subsection{Belief revision versus belief updating}

Two forms of cognitive change can be described : belief change and world change [105].

- In belief change, You hold a belief about which alternative is the actual world $\omega_{0}$ and the new information put some further constraints on which worlds can still be the actual world. This form of belief change is called a belief revision. The most classical information is that some alternatives considered as possible candidates for $\omega_{0}$ must be discarded (the conditioning process).

- In world change, evolving worlds are considered. Worlds are transformed into 'new' worlds as time changes and new information describes constraints on possible changes. This form of belief change is called a belief updating.

A typical example of the difference between belief and world changes is given by the banana/apple example. You know that a basket contains a banana or an apple or both. For belief change, let the information be that a witness looks at the basket and says there is no banana in the basket. So You conclude there is an apple in the basket. For world change, lets the information be that a banana-eater passed next to the basket and You know that the banana-eater eats any banana he sees. As in the previous case You know there is no banana in the basket. Still Your updated knowledge about the content of the basket is that the basket is either empty or contains an apple.

In belief revision the state of the world is fixed, only Your knowledge about it changes. In belief updating, the state of the world itself changes. In the TBM, belief revision is represented by the conditioning process and belief updating can be represented by the imaging process [53].

Belief revision can correspond to a static or a dynamic fusion whereas we feel belief updating corresponds always to a dynamical fusion. Consider the next examples. 
1. Belief revision. Suppose we try to classify an object. Collected data provide more and more information about the actual class. Beliefs are revised.

(a) Static fusion. Data can be collected simultaneously. We call this situation a 'static belief revision'.

(b) Dynamic fusion. Data can also be collected over time, like when classifying an approaching object in which case more recent data are probably more reliable. We call this situation a 'dynamic belief revision'.

The important issue is that the value of the variable under consideration does not change with time. In the first case, data are essentially symmetrical, whereas in the second, symmetry is not required as the more recent data are probably more reliable than the older ones.

2. Belief updating. Suppose we try to locate a moving object. The bbas will not concern the same variable, but a variable which changes over time. So each bba concerns some $X(t)$ and the actual value depends on $t$. Belief updating is not studied here.

The two types of data fusion for belief revision translate into two requirements that should be satisfied by the combination rule: associativity for static belief revision and a less demanding Markovian requirement for dynamic belief revision. We first illustrate the meaning of these two requirements.

- Associativity. Imagine You collect data from three sensors that report on the nature of an object. For logistical reasons, the reports are not delivered simultaneously. You receive the first report, represented by the bba $m_{1}$, and Your belief about the nature of the object is then represented by $m_{1}$ provide Your a priori was vacuous, i.e., represented by a vacuous belief function. Then You collect the second report, represented by the bba $m_{2}$. You combine $m_{1}$ and $m_{2}$ into a new bba $m_{12}$ that represents Your revised belief about the nature of the object. Finally You collect the third report, represented by the bba $m_{3}$, You combine it with the previous revised bba $m_{12}$. The result $m_{123}$ represents Your final belief about the nature of the object. Now suppose that You had received the reports in another order, like getting the third before the second report. You would have combined $m_{1}$ with $m_{3}$ and the result with $m_{2}$. It seems obvious that this last result should be equal to the $m_{123}$ previously considered. This requirement is the associativity requirement (section 4.2 ).

- Markovian requirement. When data are collected sequentially and time is meaningful, we may not permute the order with which data are collected. In that case, associativity is not an adequate requirement. All we need is that we can compute $m_{123}$ from $m_{12}$ and $m_{3}$, and we do not have to store the bbas that were at the origin of $m_{12}$. This requirement is called the Markovian requirement. It just means that the present belief state summarized the whole past (section 4.3).

Hidden behind these assumptions is the fact that using rules that do not comply to at least the Markovian requirement will imply the need to store all the past bbas and to recompute combinations repeatedly, in which case memory overflow and computational inefficiency are threatening the users.

We proceed by formally studying these two requirements.

\subsection{Associativity}

Associativity means that the order under which the combination is performed is irrelevant.

Let $\odot$ be any conjunctive combination operator. We remember first the definition of commutativity and associativity.

Definition 4.1 : Commutativity. The operator $\odot$ is commutative iff for any two bbas $m_{1}, m_{2}$,

$$
m_{1} \odot m_{2}=m_{2} \odot m_{1}
$$

Definition 4.2 : Associativity. The operator $\odot$ is associative iff for any three bbas $m_{1}, m_{2}, m_{3}$,

$$
\left(m_{1} \odot m_{2}\right) \odot m_{3}=m_{1} \odot\left(m_{2} \odot m_{3}\right)
$$

The binary commutative operator $\odot$ is extended into a n-ary operator as follows. 
Definition 4.3 : $\mathbf{N}$-ary $\odot$. Let $m_{1}, m_{2}, \ldots, m_{n}$ be $n$ bbas defined on $\Omega$ and let:

$$
\odot_{i=1, \ldots, n} m_{i} \triangleq f_{n}\left(m_{1}, m_{2}, \ldots, m_{n}\right) .
$$

where $f_{n}$ maps $n$ bbas into one bba.

The concept of commutativity (called then permutation symmetry) can be extended to the n-ary operator.

Definition 4.4 : Permutation symmetry. Let $m_{1}, m_{2}, \ldots, m_{n}$ be $n$ bbas defined on $\Omega$ and for every $n \geq 2$, let $f_{n}$ define a n-ary $\odot$ operator. Then $\odot$ is (permutation) symmetrical iff

$$
f_{n}\left(m_{1}, m_{2}, \ldots, m_{n}\right)=f_{n}\left(m_{i_{1}}, m_{i_{2}}, \ldots, m_{i_{n}}\right)
$$

where the $i_{j}$ 's are any permutation of the $1,2, \ldots, n$ indexes, and this property holds for any $n$.

Associativity and commutativity (permutation symmetry) imply that the order under which the data are combined with the n-ary $\odot$ operator is irrelevant as the outcome is the same. It seems a natural requirement for any static belief revision, thus any static fusion system. Among others, the Dempster's rule of combination and the TBM conjunctive combination rule are associative. Many alternate rules proposed in the literature do not satisfy associativity and therefore can be questioned.

\subsection{The Markovian requirement}

\subsubsection{No memory decay}

The Markovian requirement concerns dynamic fusion. It states that the bba computed at a given time by the combination rule should be sufficient for future combinations. Therefore past bbas are fully summarized by the present combined bba, which explains the name of the requirement.

In order to formalize this definition, suppose we collect $n$ bbas $m_{1}, m_{2}, \ldots, m_{n}$ at times $t_{1}, t_{2}, \ldots, t_{n}$ where $t_{i}>t_{i-1}$, for $i=2, \ldots n$. Let $f_{i}\left(m_{1}, \ldots, m_{i}\right)$ be the result of combining the bbas $m_{1}, \ldots, m_{i}$.

Then we want that $f_{i}\left(m_{1}, \ldots, m_{i}\right)$ is equal to a combination of $f_{i-1}\left(m_{1}, \ldots, m_{i-1}\right)$ and $m_{i}$. Let us denote this last combination by $g$, and let us assumed that $g$ is not time dependent (i.e., does not depend on $i$ ). In that case we compute iteratively:

$$
\begin{aligned}
f_{2}\left(m_{1}, m_{2}\right) & =g\left(m_{1}, m_{2}\right) \\
f_{3}\left(m_{1}, m_{2}, m_{3}\right) & =g\left(g\left(m_{1}, m_{2}\right), m_{3}\right) \\
f_{4}\left(m_{1}, m_{2}, m_{3}, m_{4}\right) & =g\left(g\left(g\left(m_{1}, m_{2}\right), m_{3}\right), m_{4}\right) \\
\text { etc... } &
\end{aligned}
$$

This property can be defined as:

Definition 4.5 : Markovian requirement. Let the bbas $m_{i}, i=1, \ldots, n$, all defined on the frame of discernment $\Omega$ and collected at times $t_{i}, i=1, \ldots, n$ with $t_{i}>t_{i-1}$, for $i=2, \ldots n$. Let $f_{n}\left(m_{1}, \ldots, m_{n}\right)$ be the bba derived by combining all the bbas $m_{1}$ to $m_{n}$. The combination represented by $f_{n}$ is qualified as Markovian iff there exists a $g$ function that combines two bbas on $\Omega$ into a bba on $\Omega$, such that:

$$
f_{n}\left(m_{1}, \ldots, m_{n}\right)=g\left(g\left(\ldots g\left(g\left(m_{1}, m_{2}\right), m_{3}\right), \ldots m_{n-1}\right), m_{n}\right) .
$$

$\mathrm{N}$-ary $\odot$ operators are too general for combination rules. Asking them to satisfy the Markovian requirement seems to grasp what dynamic combination is about. If the operator also satisfies permutation symmetry, it becomes associative, which is good for static combination, but is often asking too much for dynamic combination.

Theorem 4.1 Let a n-ary $\odot$ operator that satisfies the Markovian requirement. If $\odot$ is permutation symmetrical then it is associative. 
Proof. Let $m_{1}, m_{2}, m_{3}$ be three bbas defined on $\Omega$. Let a Markovian n-ary $\odot$ combination operator with $f_{n}$ its related function.

Suppose the n-ary operators are permutation symmetrical for all $n$. With $n=2, f_{2}\left(m_{1}, m_{2}\right)=f_{2}\left(m_{2}, m_{1}\right)$ thus $g\left(m_{1}, m_{2}\right)=g\left(m_{2}, m_{1}\right)$.

With $n=3$,

$$
\begin{aligned}
f_{3}\left(m_{1}, m_{2}, m_{3}\right) & =f_{3}\left(m_{2}, m_{3}, m_{1}\right) \\
g\left(g\left(m_{1}, m_{2}\right), m_{3}\right) & =g\left(g\left(m_{2}, m_{3}\right), m_{1}\right) \\
& =g\left(m_{1}, g\left(m_{2}, m_{3}\right)\right)
\end{aligned}
$$

hence $g$ is associative.

As $g$ is associative and commutative, the order under which the terms are combined to compute $f_{n}$ is irrelevant, in which case $f_{n}$ is associative, i.e., $f_{n}$ satisfies for all $k=1, \ldots, n-1$ :

$$
\begin{gathered}
f_{n}\left(f_{n}\left(m_{1}, \ldots, m_{n}\right), m_{n+1}, \ldots, m_{2 n-1}\right)= \\
f_{n}\left(m_{1}, \ldots, m_{k}, f_{n}\left(m_{k+1}, \ldots, m_{k+n}\right), m_{k+n+1}, \ldots, m_{2 n-1}\right)
\end{gathered}
$$

Thus for Markovian combination operators, permutation symmetry implies associativity.

\subsubsection{Memory decay}

We can add the extra requirement that 'credibility' of the beliefs themselves is decaying over time. This decaying concept can be translated by claiming that every bba is discounted with time, the longer the time since the bba was collected, the stronger the discounting. This explains why we say that memory is decaying.

So if a bba has been collected since $t$ units of time, the bba is discounted by the discount rate $\beta(t)=$ $1-\alpha(t)$ where $\alpha(t)$, the reliability factor, is a decreasing function of time with $\alpha(0)=1$ and $\lim _{t \rightarrow \infty} \alpha(t) \rightarrow 0$. When applying the $g$ function on the bbas $m_{i-1}, m_{i}$, we first discounted $m_{i-1}$ by a rate $\beta\left(d t_{i}\right)$ where $d t_{i}=t_{i}-t_{i-1}$, and combine the result with $m_{i}$.

In that case we compute iteratively:

$$
\begin{aligned}
f_{2}\left(m_{1}, m_{2}\right) & =g\left(m_{1}^{\alpha\left(d t_{2}\right)}, m_{2}\right) \\
f_{3}\left(m_{1}, m_{2}, m_{3}\right) & =g\left(g\left(m_{1}^{\alpha\left(d t_{2}\right)}, m_{2}\right)^{\alpha\left(d t_{3}\right)}, m_{3}\right) \\
f_{4}\left(m_{1}, m_{2}, m_{3}, m_{4}\right) & =g\left(g\left(g\left(m_{1}^{\alpha\left(d t_{2}\right)}, m_{2}\right)^{\alpha\left(d t_{3}\right)}, m_{3}\right)^{\alpha\left(d t_{4}\right)}, m_{4}\right) \\
\text { etc... } &
\end{aligned}
$$

where $m_{1}^{\alpha\left(d t_{2}\right)}$ is the bba that results from the discounting of $m_{1}$ by a rate $\beta_{2}\left(d t_{2}\right)=1-\alpha\left(d t_{2}\right)$, and $g\left(m_{1}^{\alpha\left(d t_{2}\right)}, m_{2}\right)^{\alpha\left(d t_{3}\right)}$ is the bba obtained by discounting the bba $g\left(m_{1}^{\alpha\left(d t_{2}\right)}, m_{2}\right)$ by a rate $\beta\left(d t_{3}\right)=1-\alpha\left(d t_{3}\right)$, etc... This property can be defined as:

Definition 4.6 : Markovian requirement with decaying memory. Let the bbas $m_{i}, i=1, \ldots, n$, all defined on the frame of discernment $\Omega$ and collected at times $t_{i}, i=1, \ldots, n$ with $t_{i}>t_{i-1}$, for $i=2, \ldots n$. Let the $f_{n}\left(m_{1}, \ldots, m_{n}\right)$ be the bba derived by combining all the bbas $m_{1}$ to $m_{n}$. Let $d t_{i}=t_{i}-t_{i-1}$, for $i=2, \ldots, n$. There exists a $g$ function that combines two bbas such that

$$
\left.f_{n}\left(m_{1}, \ldots, m_{n}\right)=g\left(g\left(\ldots g\left(g\left(m_{1}^{\alpha\left(d t_{2}\right)}, m_{2}\right)^{\alpha\left(d t_{3}\right)}, m_{3}\right)^{\alpha\left(d t_{4}\right)}, \ldots m_{n-1}\right)^{\alpha\left(d t_{n}\right)}\right), m_{n}\right) .
$$

Some constrains can be derived when using vacuous belief functions, denoted $V B F$. By lemma 2.1, the result of the discounting of the vacuous belief function is the vacuous belief function.

Suppose now we collect many vacuous belief functions while performing the dynamic combination. It is obvious that they should not interfere with the end result. Similarly, we could also consider intermediate instants where we could have collected data, but we did not do it, and we could replace the absent data by a vacuous belief function supposedly observed at these instants. End results should still be unchanged. In practice, it means that the result of the combination of a bba $m$ with a vacuous belief function is the bba $m$ itself, i.e., $g(V B F, m)=g(m, V B F)=m$. So we add the next assumption. 
Assumption 4.1 For any $m$ and $V B F$ defined on $\Omega$, the $g$ function of definition 4.6 satisfies:

$$
g(V B F, m)=g(m, V B F)=m .
$$

Under this assumption, suppose we collect the bba $m_{1}$ at time $t_{1}$, then a vacuous belief function at time $t_{2}$, and then a new bba $m_{3}$ at time $t_{3}$. The result of their combination should be the same as if we had only considered $m_{1}$ and $m_{3}$. This can be written as:

$$
g\left(g\left(m_{1}^{\alpha\left(t_{2}-t_{1}\right)}, V B F\right)^{\alpha\left(t_{3}-t_{2}\right)}, m_{3}\right)=g\left(m_{1}^{\alpha\left(t_{3}-t_{1}\right)}, m_{3}\right)
$$

By assumption 4.1, $g\left(m_{1}^{\alpha\left(t_{2}-t_{1}\right)}, V B F\right)=m_{1}^{\alpha\left(t_{2}-t_{1}\right)}$. Using lemma 2.2, the equality becomes:

$$
g\left(m_{1}^{\alpha\left(t_{2}-t_{1}\right) \alpha\left(t_{3}-t_{2}\right)}, m_{3}\right)=g\left(m_{1}^{\alpha\left(t_{3}-t_{1}\right)}, m_{3}\right)
$$

This being true whatever the bbas, the $\alpha$ function must satisfy $\alpha(t)=e^{-\gamma t}$ where $\gamma \geq 0$.

These properties are formalized in the next two lemmas.

Lemma 4.1 Let $V B F$ be a vacuous belief function, and $m$ any bba, both defined on $\Omega$. Given assumption 4.1, we have:

$$
f_{n}\left(V B F, \ldots, V B F, m_{i}, V B F, \ldots, V B F\right)=\left(\ldots\left(\left(\left(m_{i}^{\alpha\left(d t_{i}\right)}\right)^{\alpha\left(d t_{i+1}\right.}\right)^{\alpha\left(d t_{i+2}\right.}\right) \ldots\right) .
$$

Proof. Replace $V B F^{\alpha(t)}$ by $V B F$ and $g(V B F, m)$ and $g(m, V B F)$ by $m$ in relation (21).

Lemma 4.2 Given assumption 4.1, we have:

$$
g\left(g\left(m_{1}^{\alpha\left(t_{2}-t_{1}\right)}, V B F\right)^{\alpha\left(t_{3}-t_{2}\right)}, V B F\right)=m_{1}^{\alpha\left(t_{3}-t_{1}\right)}
$$

is satisfied iff $\alpha(t)=e^{-\gamma t}$ for $\gamma \geq 0$.

Proof. The requirement can be rewritten as:

$$
\begin{aligned}
g\left(g\left(m_{1}^{\alpha\left(t_{2}-t_{1}\right)}, V B F\right)^{\alpha\left(t_{3}-t_{2}\right)}, V B F\right) & =g\left(m_{1}^{\alpha\left(t_{2}-t_{1}\right)}, V B F\right)^{\alpha\left(t_{3}-t_{2}\right)} \\
& =\left(m_{1}^{\alpha\left(t_{2}-t_{1}\right)}\right)^{\alpha\left(t_{3}-t_{2}\right)} \\
& =m_{1}^{\alpha\left(t_{2}-t_{1}\right) \alpha\left(t_{3}-t_{2}\right)} \\
& =m_{1}^{\alpha\left(t_{3}-t_{1}\right)}
\end{aligned}
$$

This being true whatever $m_{1}$, we must have $\alpha\left(t_{2}-t_{1}\right) \alpha\left(t_{3}-t_{2}\right)=\alpha\left(t_{3}-t_{1}\right)$. The unique continuous solution of this Cauchy equation is given by $\alpha(t)=e^{-\gamma t}[1]$. The limit conditions $\alpha(0)=1$, and $\lim _{t \rightarrow \infty} \alpha(t) \rightarrow 0$ are satisfied iff $\gamma \geq 0$.

These properties do not impose strong requirements on $g$, except $g(V B F, m)=g(m, V B F)=m$ which seems unavoidable.

In the TBM, the Markovian property with decaying memory has also the nice property that if at a given instant, there is a full conflict, i.e., $m(\emptyset)=1$, the next data are nevertheless not fully absorbed by it, and after enough time the system will automatically recover, as the memory of the conflictual bba decays with time. Without memory decay, the TBM would stay forever in a state of full conflict.

Table 1 presents an example of application of the dynamic fusion based on the TBM conjunctive combination rule with decaying memory. At time $t_{2}=3$, the 'disc. old bba' is obtained by discounting the bba collected at time $t_{1}=1$ (using $\gamma=0.15$ ). The revised bba is obtained by applying the TBM conjunctive combination rule on the just computed discounted bbas and the new bba collected at that instant. The BetP column gives the pignistic probabilities for the revised bba. 


\begin{tabular}{|c|c|c|c|c|c|c|}
\hline time & $d t$ & power set & new bba & disc. old bba & revised bba & BetP \\
\hline \multirow[t]{8}{*}{$t_{1}=1$} & & $\emptyset$ & 0 & & 0 & \\
\hline & & $a$ & 0.3 & & 0.3 & 0.55 \\
\hline & & $b$ & 0 & & 0 & 0.35 \\
\hline & & $c$ & 0 & & 0 & \\
\hline & & $a, b$ & 0.5 & & 0.5 & \\
\hline & & $a, c$ & 0 & & 0 & \\
\hline & & $b, c$ & 0.2 & & 0.2 & \\
\hline & & $a, b, c$ & 0 & & 0 & \\
\hline \multirow[t]{8}{*}{$t_{2}=3$} & 2 & $\emptyset$ & 0 & 0 & 0.056 & \\
\hline & & $a$ & 0 & 0.222 & 0.389 & 0.54 \\
\hline & & $b$ & 0.25 & 0 & 0.217 & 0.28 \\
\hline & & $c$ & 0 & 0 & 0.089 & 0.18 \\
\hline & & $a, b$ & 0.15 & 0.370 & 0.094 & \\
\hline & & $a, c$ & 0.60 & 0 & 0.155 & \\
\hline & & $b, c$ & 0 & 0.148 & 0 & \\
\hline & & $a, b, c$ & 0 & 0.259 & 0 & \\
\hline \multirow[t]{8}{*}{$t_{3}=4$} & 1 & $\emptyset$ & 0 & 0.048 & 0.360 & \\
\hline & & $a$ & 0.70 & 0.335 & 0.524 & 0.84 \\
\hline & & $b$ & 0.20 & 0.186 & 0.081 & 0.13 \\
\hline & & $c$ & 0 & 0.077 & 0.008 & 0.03 \\
\hline & & $a, b$ & 0 & 0.081 & 0 & \\
\hline & & $a, c$ & 0.10 & 0.134 & 0.027 & \\
\hline & & $b, c$ & 0 & 0 & 0. & \\
\hline & & $a, b, c$ & 0 & 0.139 & 0 & \\
\hline
\end{tabular}

Table 1: Dynamic combination of bbas with a memory decay. Combination performed with the TBM conjunctive combination rule. Discounting rate with $\gamma=0.15$. Columns are the times $(1,3$ and 4 time units) at which bbas are collected, the time delays between two successively collected bbas, the elements of the power sets, the collected bbas, the result of discounting the past bbas, the combination of past and present bbas, its pignistic transformation. Discount rates are 0.741 at $t_{2}=3$ and 0.861 at $t_{3}=4$. 


\section{The assumptions behind the combination rules}

The first thing to do when encountering a conflict consists in verifying if the applicability criteria for a combination are satisfied. This can be achieved by an expert systems that analyzes the origin of the conflict between bbas and takes appropriate decisions on how to combine them. Section 6 concentrates on this issue.

Before studying such an expert system, we must determine what are the assumptions that underlie the application of the combination rule. This analysis applies of course to the TBM conjunctive combination rule, but also to most systems for belief combination as far as most of them depend on the same conjunctive rule (see section 1, relation (1)).

Amazingly, authors who develop new combination rules hardly discuss about the fact that maybe the combination prerequisites are unsatisfied. One must at least consider if the pieces of evidence that underlie the bbas are indeed distinct. Failing to perform such a verification is as erroneous as accepting by default stochastic independence in probability theory. It seems some authors even accept uncritically the idea that Dempster-Shafer theory provides a systematic uncertainty management system that makes other uncertainty management systems unnecessary. The same assertion has also been made as uncritically for logic, probability theory, fuzzy sets theory, possibility theory, just to name a few. Things are more subtle, and before using a model for uncertainty, one must study the nature of the uncertainty and of the imprecision that pervade the data [97].

The assumptions underlying the conjunctive rules concern the sources reliability, the distinctness of the bbas to be combined, the equality of the referred objects, the closed world assumption, and a parsimony property. The first is related to source rating whereas the others concern the report rating (see section 3.7).

We then show that the conflict can also be understood as the belief that the hypothesis of object equality is false (section 5.2). Resolving the conflict will be studied in the next section (section 6).

\subsection{The assumptions}

In order to understand what to do with the weight of conflict $k$ (or $m(\emptyset)$ ), we examine exactly what are the assumptions that underlie the applicability of the TBM conjunctive combination rule.

Let $S_{1}$ be a source of information. It observes an object $o_{1}$ and produces a bba $m_{1}^{\Omega}\left\{o_{1}\right\}$ expressing its opinion about the actual value $\omega_{01}$ of a variable $X$ that depends on $o_{1}$, where $\omega_{01} \in \Omega$.

Let $S_{2}$ be a source of information. It observes an object $o_{2}$ and produces a bba $m_{2}^{\Omega}\left\{o_{2}\right\}$ expressing its opinion about the actual value $\omega_{02}$ of a variable $X$ that depends on $o_{2}$, where $\omega_{02} \in \Omega$.

You collect these two bbas and want to combine them. For $i=1,2$, let $m_{Y}^{\mathcal{R}}\left\{S_{i}\right\}$ be Your bba about the fact that source $S_{i}$ is reliable or not, with $\mathcal{R}=\{$ Reliable, not_Reliable $\}=\{R, N R\}$.

The assumptions used for applying the TBM conjunctive combination rule are listed hereafter.

Assumption 5.1 : $R S 1 . S_{1}$ is fully reliable.

It can be translated as $m_{Y}^{\mathcal{R}}\left\{S_{1}\right\}(R)=1$. It means among others that if You had only collected $S_{1}$ data, Your belief about the actual value of $\Omega$ for object $o_{1}$ would be equal to the one collected from $S_{1}$ :

$$
m_{Y}^{\Omega}\left\{o_{1}\right\}\left[m_{1}^{\Omega}\left\{o_{1}\right\}, R S 1\right]=m_{1}^{\Omega}\left\{o_{1}\right\} .
$$

This assumption can be extended by accepting that $S_{1}$ is partially reliable. This would mean that Your bba would be equal to the result of discounting $S_{1}$ 's bba. This partial reliability generalization is not used here.

Assumption 5.2 : $R S 2 . S_{2}$ is fully reliable.

As above, it can be translated as $m_{Y}^{\mathcal{R}}\left\{S_{2}\right\}(R)=1$ and we have:

$$
m_{Y}^{\Omega}\left\{o_{2}\right\}\left[m_{2}^{\Omega}\left\{o_{2}\right\}, R S 2\right]=m_{2}^{\Omega}\left\{o_{2}\right\} .
$$

Assumption 5.3 : Dist. The two bbas $m_{1}^{\Omega}\left\{o_{1}\right\}$ and $m_{2}^{\Omega}\left\{o_{2}\right\}$ are based on distinct pieces of evidence. 
Given assumptions $R S 1, R S 2$ and Dist, You can build Your joint bba on the actual values of the two objects (we have not yet said the two objects are the same object).

$$
m_{Y}^{\Omega \times \Omega}\left\{o_{1}, o_{2}\right\}\left[m_{1}^{\Omega}\left\{o_{1}\right\}, m_{2}^{\Omega}\left\{o_{2}\right\}, R S 1, R S 2, \text { Dist }\right]=m_{1}^{\Omega}\left\{o_{1}\right\} @ m_{2}^{\Omega}\left\{o_{2}\right\}
$$

where for all $A \subseteq \Omega \times \Omega$.

$$
m_{1}^{\Omega}\left\{o_{1}\right\} @ m_{2}^{\Omega}\left\{o_{2}\right\}(A)= \begin{cases}m_{1}^{\Omega}\left\{o_{1}\right\}(B) m_{2}^{\Omega}\left\{o_{2}\right\}(C) & \text { if } A=(B, C), B, C \subseteq \Omega, \\ 0 & \text { otherwise }\end{cases}
$$

The product rule results from the doxastic independence assumed by Dist (see section 3.1). It is the analogous of the product rule for probability measures used under stochastic independence.

Assumption $5.4: E q O$. Objects $o_{1}$ and $o_{2}$ are the same object.

The assumption $E q O$ can be represented as:

$$
m_{Y}^{\Omega \times \Omega}\left\{o_{1}, o_{2}\right\}(E q)=1
$$

where $E q$ is the subset $\left\{\left(\omega_{1}, \omega_{2}\right): \omega_{1}=\omega_{2}\right\} \subseteq \Omega \times \Omega$ (those elements of $\Omega \times \Omega$ where the two values are equal) and $\overline{E q}$ denotes its complement relative to $\Omega \times \Omega$.

Deriving the TBM conjunctive combination rule. Adding assumption $E q O$ to $R S 1, R S 2$ and Dist means conditioning the bba of relation (22) on $E q$ by the unnormalized Dempster's rule of conditioning, and marginalizing the result on $\Omega$ which is in one to one relation with $E q$ ( $\omega \in \Omega$ is the element of $\Omega$ that corresponds to the element $(\omega, \omega) \in E q)$. One gets for all $A \subseteq \Omega$ :

$$
\begin{aligned}
m_{Y}^{\Omega}(A) & =m_{Y}^{\Omega \times \Omega}\left\{o_{1}, o_{2}\right\}[E q](\{(\omega, \omega): \omega \in A\}) \\
& =\sum_{B, C \subseteq \Omega: B \cap C=A} m_{1}^{\Omega}\left\{o_{1}\right\}(B) m_{2}^{\Omega}\left\{o_{2}\right\}(C)
\end{aligned}
$$

This derivation fits with the one described in [79, page 58].

As can be seen from this relation, this is what we get when applying the TBM conjunctive combination rule on $m_{1}^{\Omega}$ and $m_{2}^{\Omega}$ (provided $E q O$ is accepted):

$$
m_{Y}^{\Omega}=m_{1}^{\Omega} @ m_{2}^{\Omega} .
$$

We introduce now a strong form of closed world assumption, denoted $(C w a)$.

Assumption 5.5 : Cwa. The frame of discernment is necessarily exhaustive.

When discussing the nature of the frame of discernment $\Omega$ of a bba $m^{\Omega}$, we feel that in general one should consider that $\Omega$ is not exhaustive. This can easily occur in practice when You build $\Omega$ as a list of possible alternatives, of possible worlds. It is obvious that some alternatives can always be forgotten. In that case, we say that we work under the open world assumption.

More surprisingly, open world can also be considered with dice tossing experiments. We consider the 'heads' worlds and the 'tails' worlds. And what if the coin disintegrates in the space, falls on the hedge, violates all gravity laws and goes up for ever...

All this just shows that we can always face problems with exhaustively (i.e., with the closed world assumption) and there does not seem to exist an obvious solution. We already discuss in section 3.5 the idea to add an extra alternative that covers all the alternative not considered in $\Omega$, but emphasize that this solution is not obvious in belief function theory. This is why we develop the TBM in an open world framework... and accept $m(\emptyset)>0$. We feel presumptuous to assume that $\Omega$ is necessarily exhaustive, thus to assume to be working in a closed world framework.

Nevertheless if You can defend the closed world assumption, Your beliefs derived under assumptions 5.1 to 5.4 as $m_{1}^{\Omega} @ m_{2}^{\Omega}$ by the application of the TBM conjunctive combination rule can now be normalized, in which case the TBM conjunctive combination rule is replaced by Dempster's rule of combination: Under Cwa (and assumptions 5.1 to 5.4 ), $m_{Y}^{\Omega}=m_{1}^{\Omega} \oplus m_{2}^{\Omega}$.

We finally add a parsimony principle that is necessary, even thought often neglected.

Assumption 5.6 : Pars. Keep as many assumptions as possible.

It will be accepted in every case analyzed hereafter. 


\subsection{Belief on $\{E q, \overline{E q}\}$}

Consider the frame $\mathfrak{E q}=\{E q, \overline{E q}\}$ ( $\mathfrak{E q}$ is a coarsening of $\Omega \times \Omega)$ and $E q=\left\{\left(\omega_{1}, \omega_{2}\right): \omega_{1}=\omega_{2}\right\}$ and $\overline{E q}$ being the complement of $E q$ relative to $\Omega \times \Omega$.

Let $m^{\mathfrak{E} \mathfrak{q}}$ be the result of coarsening $m_{Y}^{\Omega \times \Omega}\left\{o_{1}, o_{2}\right\}$ on $\mathfrak{E q}$. We have [19]:

$$
\begin{aligned}
m^{\mathfrak{E q}}(E q) & =\sum_{\omega \in \Omega} m_{Y}^{\Omega \times \Omega}\left\{o_{1}, o_{2}\right\}(\omega, \omega) \\
& =\sum_{\omega \in \Omega} m_{1}^{\Omega}\left\{o_{1}\right\}(\omega) m_{2}^{\Omega}\left\{o_{2}\right\}(\omega), \\
m^{\mathfrak{E q}}(\overline{E q}) & =\sum_{\omega_{1}, \omega_{2} \subseteq \Omega, \omega_{1} \cap \omega_{2}=\emptyset} m_{Y}^{\Omega \times \Omega}\left\{o_{1}, o_{2}\right\}\left(\omega_{1}, \omega_{2}\right) \\
& =\sum_{\omega_{1}, \omega_{2} \subseteq \Omega, \omega_{1} \cap \omega_{2}=\emptyset} m_{1}^{\Omega}\left\{o_{1}\right\}\left(\omega_{1}\right) m_{2}^{\Omega}\left\{o_{2}\right\}\left(\omega_{2}\right), \\
m^{\mathfrak{E q}}(\emptyset) & =m_{Y}^{\Omega \times \Omega}\left\{o_{1}, o_{2}\right\}(\emptyset) \\
& =m_{1}^{\Omega}\left\{o_{1}\right\}(\emptyset)+m_{2}^{\Omega}\left\{o_{2}\right\}(\emptyset)-m_{1}^{\Omega}\left\{o_{1}\right\}(\emptyset) m_{2}^{\Omega}\left\{o_{2}\right\}(\emptyset), \\
m^{\mathfrak{E q}}(\mathfrak{E q}) & =1-m^{\mathfrak{E q}}(E q)-m^{\mathfrak{E q}}(\overline{E q})-m^{\mathfrak{E q}}(\emptyset) .
\end{aligned}
$$

Suppose we had accepted $E q O$. Let $m_{i}=m_{i}^{\Omega}\{o\}$ for $i=1,2$. Suppose we compute $m_{1} @ m_{2}$. We have $m_{1} @ m_{2}(\emptyset)=m^{\mathfrak{E q}}(\overline{E q})+m^{\mathfrak{E q}}(\emptyset)$.

The term $m^{\mathfrak{E q}}(\overline{E q})$ is the mass $m_{1} @ m_{2}(\emptyset)$ when both $m_{1}$ and $m_{2}$ are normalized. With unnormalized bbas, it is the part of $m_{1} @ m_{2}(\emptyset)$ that results from an inherent conflict between $m_{1}$ and $m_{2}$.

The term $m^{\mathfrak{E q}}(\emptyset)$ is that part of $m_{1} @ m_{2}(\emptyset)$ that is related to preexisting conflicts, i.e., conflicts already present in $m_{1}$ and $m_{2}$.

Thanks to this bba on $\mathfrak{E q}$, the mass $m_{1} @ m_{2}(\emptyset)$ gets here a perfect explanation [19]. When $m_{1}$ and $m_{2}$ are normalized, the mass $m_{1} @ m_{2}(\emptyset)$ is the belief that the two sources report beliefs about different objects. Indeed $b e l^{\mathfrak{E q}}(\overline{E q})=m^{\mathfrak{E q}}(\overline{E q})$. When $m_{1}$ and $m_{2}$ are not normalized, the second component reflects the conflict that was already present in the two bbas. With such an explanation for the meaning of the mass given to the empty set, there is no more a necessity to ask for rules that renormalize the bbas after combination.

\section{Resolving conflict in the TBM}

Suppose now we face a (maybe large) conflict when trying to combine two belief functions. What should we do to resolve this apparently problematic situation. In the TBM, the conclusions are clear, keep the conflict on the empty set, don't hide it, and use an expert system to resolve it when feasible (section 6.1). It means in particular to reconsider all the assumptions that underlie the application of the combination rules (section 6.2). Still, some authors might be unhappy with that solution, so we present some other solutions, even though personally we do not support them (section 6.3).

\subsection{The expert system for resolving conflict}

In the TBM, the presence of a mass on the empty set is an alarm to indicate that there is a conflict between the two bbas. This should trigger an expert system that would study the origin of the bbas, the nature of the sources and of the pieces of evidence used. Such expert system has nothing to do with belief functions themselves. One can discover that one of the bbas needs to be discounted, or that the equality of the two object must be reconsidered.

The very successful anti-personal mine detection system $[62,61,63,60]$ is based on an expert system that handles the discounting of the bbas generated by the about 25 sources.

The system for clustering thousands of sources according to which object they report about $[71,72,73$, $74,3]$ is fully based on the analysis of the conflict by an expert system that tries to associate objects and bbas by minimizing the conflicts between the bbas reporting on the same object. 
The association problem for deciding which object at a given time corresponds to which object at another time is also solved by an expert system analyzing the conflicts [68].

In [55], the author suggests a measure of conflict where $m(\emptyset)$ is one part of the criteria, the other being based on the difference between the pignistic probabilities.

There still remains one maybe embarrassing case. Suppose You have no a priori whatsoever about both sources and for simplicity sake each source communicates a categorical bba. Suppose the bbas are fully conflictual $(k=1)$. None of the expert systems can resolve the conflict as You are in a state of perfect symmetry. What to do? Answering that problem would mean we can answer to the same logical issue when both propositions $p$ and $\neg p$ are true. Solving the conflict encountered with belief functions is like solving this logical contradiction, still an open problem after more than two thousand years of logic. In fact all You can conclude is that there is conflict and You do not know its origin. Normalizing would just mean hiding the issue.

This expert system is of course strongly application dependent. We can only present guidelines on the properties that should be analyzed and hints on what to do.

Fgure 1 presents a flow chart that might be used for implementing such an expert system. Details about its nodes are given in the next section. The queries in the diamonds are :

1. Disc Inf $=$ Do You have some information about the corresponding sensor reliability?

2. Dist $=$ The two pieces of evidence are distinct?

3. $E q O=$ The two objects are equal?

4. Close $\Omega=$ Do You want to close $\Omega$ by adding an extra alternative $\omega^{*}$ ?

$5 . \quad k$ tol $=$ Do You consider the amount of conflict $k$ tolerable?

6. Tune $\alpha=$ Do You want to tune the $\alpha_{1}$ and $\alpha_{2}$ coefficients?

7. Rej one $R s_{i}=$ Do You want to reject one of $R S 1$ or $R S 2$ ?

8. Rej $E q O=$ Do You want to reject the equal object assumption?

9. Fix $k=$ Do You want to find a rule that fix $k$ ?

\subsection{Eliminating conflict by rejecting one assumption}

We analyze what would be the impact of rejecting each of the assumptions of section 5.1 one by one. Table 2 summarizes the results detailed hereafter.

\begin{tabular}{|c|c|c|c|}
\hline Case & Rejected assumption & Combi. & Rule \\
\hline $\begin{array}{l}1 \mathrm{a} \\
1 \mathrm{~b} \\
1 \mathrm{c} \\
2 \\
3 \\
4\end{array}$ & $\begin{array}{l}R S 1 \text { or } R S 2 \text { not OK } \\
\text { - partial discount } \\
\text { - full discount: OR } \\
\text { - one lies: XOR } \\
\text { Dist } \\
\text { EqO } \\
\text { Cwa }\end{array}$ & $\begin{array}{c}m_{1}^{\alpha_{1}} @ m_{2}^{\alpha_{2}} \\
m_{1} @ m_{2} \\
m_{1} \bigcup m_{2} \\
m_{Y}^{\Omega \times \Omega}\left\{o_{1}, o_{2}\right\} \\
2 \text { objects } \\
\omega_{0} \notin \Omega\end{array}$ & $\begin{array}{l}\text { tolerable conflict } \\
\text { disjunctive combi. } \\
\text { exclusive or } \\
\text { extra information } \\
\text { Schubert } \\
\text { TBM conj. combi. }\end{array}$ \\
\hline & Accept assumptions & & \\
\hline $\begin{array}{l}5 \\
6 \\
7 \\
8 \\
\end{array}$ & $\begin{array}{l}\text { mass by mass } \\
\text { average }+\mathrm{VBF} \\
\text { average }+ \text { contr } \\
\text { register conflict }\end{array}$ & $\begin{array}{c}\text { mix } \cap \text { and } \cup \\
\text { put } k \text { in } \Omega \\
\text { allocate } k \text { on unions } \\
\text { leave } k \text { on } \emptyset\end{array}$ & $\begin{array}{l}\text { Dubois and Prade } \\
\text { Yager } \\
\text { TBM conj. combi. } \\
\text { TBM conj. combi. }\end{array}$ \\
\hline
\end{tabular}

Table 2: Rules resulting from the rejection of one of the assumptions of section 5.1 or from their acceptation. $k$ denotes the mass given to the empty set by the TBM conjunctive combination rule, i.e., the conflict. 


\subsubsection{Rejecting $R S 1$ or $R S 2$}

One solution consists in rejecting at least one of $R S 1$ and $R S 2$. It means discounting the corresponding bba. The case for rejecting both $R S 1$ and $R S 2$ is not considered because of the Pars assumption 5.6.

The issue is to decide which bba to discount and by how much. When there are many bbas, one can introduce some majority rules (see section 6.4). One can also use a stepwise procedure to eliminate (fully discounted) the bbas which are in high conflict with the other bbas (see appendix A.6).

Of course with only two bbas such elimination consists in eliminating the one with the largest mass given to $\emptyset$. But as we can expect normalized bbas, the procedure will not help as both masses are null. In such symmetrical cases and without any external information about the source reliability, such selective elimination procedure cannot be applied. You can then apply one of the next three methods.

1. You can discount both bbas at a rate large enough so that the conflict between the discounted bbas becomes 'tolerable' [3] (case 1a in table 2).

2. You can fully discount the report of one of the sources, without knowing which one. In that case, You apply the disjunctive combination rule (case $1 \mathrm{~b}$ in table 2).

3. You can claim that one of the sources lies, but You don't know which one [103]. In that case, You apply the 'exclusive or' rule (see relation (13) (case 1c in table 2).

The choice between these three methods depend on which of them can be defended. It is of course problem dependent.

\subsubsection{Rejecting Dist}

It means we reject that the two bbas are produced by distinct pieces of evidence. In that case the combination is no more derivable from its marginals, and You must provide explicitly the joint belief on the product space $\Omega \times \Omega$ for the pair $\left\{\omega_{01}, \omega_{02}\right\}$.

\subsubsection{Rejecting $E q O$}

Another solution consists in rejecting the assumption $E q O$. In that case, we must decide how many objects are under consideration, and which sources are reporting on which objects. Combination is performed on those bbas related to the same objects. This is what Schubert's method advices [70, 71] (case 3 in table 2). The issue is to decide when conflicts are too large to accept that the number of object is correctly assessed. Justifying a hard rule seems hopeless. Being 'too large' is a fuzzy concept, and must be handled as such. A suggestion on tolerable conflict is given in [3], but other solutions could as well be defended [55].

\subsubsection{Rejecting $C w a$}

Suppose both $m_{1}$ and $m_{2}$ are normalized as if their sources accept that $\Omega$ is exhaustive. Why do You have to accept this claim? If You remove the exhaustivity, You an redefine $\Omega$ and create $\Omega^{*}=\Omega \cup\left\{\omega^{*}\right\}$. You reconsider the collected bbas defined on $\Omega$ and vacuously extend them on $\Omega^{*}$, what means that for all $A \subseteq \Omega^{*}: m^{\Omega^{*}}(A)=m^{\Omega}(B)$ if $A=B \cup\left\{\omega^{*}\right\}$ and 0 otherwise. This reallocation of the masses fits with the least commitment principle, but other reallocations could be considered (see section 3.5).

After these belief extensions, conflict disappears as $\omega^{*}$ belongs to every focal element of $m_{1}$ and $m_{2}$. All masse after combination are transferred to the set extended with $\omega^{*}$ and the mass allocated initially at $\emptyset$ is given now to $\omega^{*}$.

Of course if we then condition the result on $\Omega$, we are back to the TBM conjunctive combination rule with its conflict allocated to the empty set (case 4 in table 2 ).

\subsubsection{Rejecting Pars}

Once the Pars assumption is abandoned, we can apply several of the previous suggestions simultaneously. For instance, we can then reject both $R S 1$ and $R S 2$, in which case Your combined belief is just a vacuous belief function. Pars could be rejected when the conflict cannot be resolved by relaxing one assumption. 


\subsection{Normalizing the bbas while accepting all assumptions}

For those who still want to normalize the results of the combination and to keep all the previous assumptions, we can consider (at least) two ways to perform the combination when there is some conflict. In this section, which focuses on normalizing bbas, both $m_{1}$ and $m_{2}$ are normalized bbas.

\subsubsection{Solution one mass at a time.}

Consider each product $m_{1}^{\Omega}(A) m_{2}^{\Omega}(B)$. It can be understood as the weight given to the categorical case where $A$ and $B$ holds. You combine these $A$ and $B$ by union if $A \cap B=\emptyset$ or intersection if $A \cap B \neq \emptyset$ and transfer the weight $m_{1}^{\Omega}(A) m_{2}^{\Omega}(B)$ to it. You add the weights transferred to the same sets of $\Omega$. The end result is Your bba on $\Omega$ obtained after combining the bbas reported by the two sources given You accept the assumptions $R S 1, R S 2$, Dist and $E q O$. The final bba is given for all $A \subseteq \Omega, A \neq \emptyset$ by:

$$
m_{Y}^{\Omega}(A)=\sum_{B, C \subseteq \Omega, B \cap C=A} m_{1}^{\Omega}(B) m_{2}^{\Omega}(C)+\sum_{B, C \subseteq \Omega, B \cap C=\emptyset, B \cup C=A} m_{1}^{\Omega}(B) m_{2}^{\Omega}(C)
$$

and $m_{Y}^{\Omega}(\emptyset)=0$. This is Dubois and Prade's rule (case 5 in table 2).

\subsubsection{Solution one bba with one bba.}

Consider each bba as (generalized weighted) sets, build their union (disjunctive combination rule) and intersection (TBM conjunctive combination rule), and 'average' these results ${ }^{6}$.

The union of the two normalized bbas $m_{1}$ and $m_{2}$ is given by the disjunctive combination rule denoted by the (1) operator. The intersection of the two bbas $m_{1}$ and $m_{2}$ assuming the closed world assumption is given by Dempster's rule of combination denoted by $\oplus$. The intersection of the two bbas $m_{1}$ and $m_{2}$ without assuming the closed world assumption is given by TBM conjunctive combination rule denoted by (D).

Suppose $m_{1} @ 2(\emptyset)$ is positive. Remember (see section 5.2) that $m^{\mathfrak{E q}}(\overline{E q})=m_{1} @ 2(\emptyset)$, and the denominator of Dempster's rule of combination is $1-m_{1} @ 2(\emptyset)=p l^{\mathfrak{E q}}(E q)$.

We focus now on the meaning of the masses of $m^{\mathfrak{E q}}$. If $m^{\mathfrak{E q}}(\overline{E q})$ is positive, You face the need to create a 'combination rule' that can be applied when $E q$ does not hold, thus when the objects about which $m_{1}$ and $m_{2}$ report are different. Several suggestions for such a 'combination rule' leads to some of the well known normalization methods, what may cast some light on their origin.

- The mass $m^{\mathfrak{E q}}(E q)$ supports that $E q$ holds. It means that all assumptions $R S 1, R S 2$, Dist and $E q O$ are compatible, in which case Your belief on $\omega_{0}$ is given by $m_{1}^{\Omega} @ 2=m_{1}^{\Omega} @ m_{2}^{\Omega}$ or $m_{1 \oplus 2}^{\Omega}=m_{1}^{\Omega} \oplus m_{2}^{\Omega}$ (it depends on the open or the closed world assumption).

- The mass $m^{\mathfrak{E} q}(\mathfrak{E q})$ can support both that $E q$ or $\overline{E q}$ holds. In the second case, assumption $E q O$ does not hold, whereas in the first it does. By the parsimony principle, we select the case were $E q O$ holds, and thus this mass supports the same solution as the previous case.

- The mass $m^{\mathfrak{E} \mathfrak{q}}(\overline{E q})$ supports that $E q$ does not hold, thus You should somehow fix the assumptions. Let the still to be defined solution be temporarily denoted by $m_{1 ? 2}^{\Omega}$ where the operator ? emphasizes its still undefined nature.

- The mass $m^{\mathfrak{E q}}(\emptyset)=0$ as both bbas $m_{1}$ and $m_{2}$ are normalized.

The bba $m_{Y}^{\Omega}$ generated by the combination of these components, and using $\oplus$ is given by:

$$
m_{Y}^{\Omega}=\left(1-m^{\mathfrak{E} \mathfrak{q}}(\overline{E q})\right) m_{1 \oplus 2}^{\Omega}+m^{\mathfrak{E} \mathfrak{q}}(\overline{E q}) m_{1 ? 2}^{\Omega},
$$

which can also be written as:

$$
m_{Y}^{\Omega}(A)= \begin{cases}m_{1}^{\Omega} @_{2}(A)+m_{1}^{\Omega} @_{2}(\emptyset) m_{1 ? 2}^{\Omega}(A) & \forall A \subseteq \Omega, A \neq \emptyset \\ m_{1 @ 2}^{\Omega}(\emptyset) m_{1 ? 2}^{\Omega}(\emptyset) & \text { if } A=\emptyset\end{cases}
$$

Several solutions can be considered for $m_{1 ? 2}^{\Omega}$.

${ }^{6}$ Averaging is perfectly justified within the TBM [92]. 
- Use $m_{1 ? 2}^{\Omega}=m_{1}^{\Omega}()_{2}$, the disjunctive combination of $m_{1}^{\Omega}\left\{o_{1}\right\}$ and $m_{2}^{\Omega}\left\{o_{2}\right\}$ assuming $E q O$ (i.e., $o_{1}=o_{2}$ ). The final bba is:

$$
m_{Y}^{\Omega}(A)= \begin{cases}m_{1 @ 2}^{\Omega}(A)+m_{1 @ 2}^{\Omega}(\emptyset) m_{1()_{2}}^{\Omega}(A) & \forall A \subseteq \Omega, A \neq \emptyset \\ m_{1 @ 2}^{\Omega}(\emptyset) m_{1()_{2}}^{\Omega}(\emptyset) & \text { if } A=\emptyset\end{cases}
$$

This seems to be a new rule. It is special form of the unified combination rule given by relation (28).

- Consider that You are lost as the assumptions are inconsistent, and therefore You reserve Your opinion. This can be understood to mean that You are in a state of full ignorance, in which case Your belief is represented by is a vacuous belief function. In that case, your final bba is given by:

$$
m_{Y}^{\Omega}(A)= \begin{cases}m_{1 @ 2}^{\Omega}(A) & \forall A \subseteq \Omega, A \neq \emptyset, A \neq \Omega \\ m_{1 @ 2}^{\Omega}(\Omega)+m_{1}^{\Omega} \varrho_{2}(\emptyset) & \text { if } A=\Omega \\ 0 & \text { if } A=\emptyset\end{cases}
$$

This is Yager's rule (case 6 in table 2).

- Consider that You are lost as the assumptions are inconsistent. You acknowledge it by accepting a full contradiction bba, thus a bba with a mass 1 on $\emptyset$ in which case Your belief is represented by a contradictory belief function. In that case, your final bba is given by:

$$
m_{Y}^{\Omega}=m_{1 @ 2}^{\Omega}
$$

This is the TBM conjunctive combination rule (case 7 in table 2).

Which of the three solutions is adequate is unclear. We advocate the third but present the other twos to show how they fit in this more general framework.

\subsection{The need for an uncertainty management system}

As illustrated by the many suggestions, there is no obvious solution to the issue of normalization. Many doors have been opened, but none seems the winner for solving every problem.

We feel that the best solution is the creation of some context dependent expert systems that detects the conflict issue and when necessary solves it by finding its origin.

A criticism addressed against the TBM conjunctive combination rule is based on the absorbing effect of a full contradictory bba. Once $m(\emptyset)=1$, its combination with any other bba results always in a full contradictory belief function. This case is not supposed to occur as encountering $m(\emptyset)=1$ must switch the expert system on and its outcome should restore a non conflictual bba. Nevertheless it could occur, and we must consider what to do in such a case.

Suppose the simplified case where we have $r$ categorical bbas with $m_{i}(A)=1, i=1, \ldots, r$, and $s$ categorical bbas with $m_{i}(B)=1, i=r+1, \ldots, r+s$, and $A \cap B=\emptyset$.

Two cases can be considered.

Eliminating one bba. Consider first the case with $s=1$ and $r$ large. Given the conflict, the expert system is switch on, and the stepwise algorithm for the elimination of conflictual bbas (see Appendix A.6) will detect that the source of the conflict is $m_{r+1}$. This bba will then be fully discounted (what is equivalent to discarding it), and coherence will be recovered.

Rejecting $E q O$. Consider next the case where both $r$ and $s$ are larger, like $5,10, \ldots$ Given the conflict, the expert system is switch on, the plain elimination of the conflictual bbas seems not adequate. Indeed, how could one explain that so many sensors report $A$ and about the same number report $B$. Common sense dictates that the sensors do not report on the same object. Hence $E q O$ assumption is not valid. Sensors must be clustered according to the objects they each report about. An efficient algorithms applicable to thousands sensors and several dozens objects is presented in [73, 75, 77].

It is obvious that there is no way to represent inside the categorical bbas the fact there were 10 or 20 sources that support $A$. In both cases, the resulting bba is the same categorical one focused on $A$. This 
issue enhances the need for an uncertainty management system which stores external information linked to every bba. The bbas are not sufficient to represent the whole belief state. They represent the beliefs held by the agents, but the agents must also store information about the sources and the reports themselves. In particular the numbers $r$ and $s$ are obviously to be stored. What information must be stored in the uncertainty management system is application dependent and out of the scope of the present paper (see $[25,26])$.

\section{Conclusions}

The existence of a (large) conflict when combining several basic belief assignments and Zadeh's counterexample have lead to many publications where authors propose solutions that too often turn out to be ad hoc, and create confusion among the users of the belief function theory.

We reexamine the issue comparing the Dempsterian models which are based on an underlying probability model and the transferable belief model where no underlying probability model is assumed. We enhance that Dempster's rule of combination is justified in the Dempsterian models whereas the TBM conjunctive combination rule is justified in the TBM. Many other suggestions have been proposed, but most lack such kind of formal justifications.

In the TBM, the existence of a conflict is not really an issue. It is allocated to the $\emptyset$ and corresponds to at least three sources, the fact we work under the open world assumption, the existence of conflict between the combined bbas, and the belief that the two bbas do not report on the same object. A large conflict justifies that an expert system examines its origin and takes the appropriate measure. This property is at the core of several highly successful real life applications of the TBM.

For those who really want a normalized combination (that is not asked for in the TBM), we examine what are the assumptions that underlie the combination rules. We determine how to resolve conflict either while keeping these assumptions, or by relaxing them.

We hope this paper will have clarified the issue of the conflictual combination in the belief function theory.

Finally we would like to mention that the lack of normalization can be computationally dangerous. Indeed after many combinations, the mass given to the empty set often becomes close to 1, and rounding errors can occur. So even if we advice keeping track of the conflict, we also advice some partial renormalization when implementing belief function based algorithms. This partial renormalization is of course just a computational trick, and has nothing to do with the underlying conceptual problem.

\section{Appendix A.}

\section{A.1 Doxastic independence}

In $[5,6]$, the concept of doxastic independence between two variables is introduced and defined as irrelevance plus irrelevance preservation.

- Irrelevance: beliefs about one variable does not influence the beliefs about the second, and

- Irrelevance preservation under the TBM conjunctive combination rule ${ }^{7}$ : if we combine two bbas defined on the product space of two variables that satisfy irrelevance, the resulting bba still satisfies irrelevance.

Authors then show that two variables are doxastically independent if and only if the joint bba can be computed from its two marginals by applying the TBM conjunctive combination rule to build a bba on a product space (this property is called 'non interactivity'). This result is not obtained if one only assumes irrelevance. Assimilating distinctness with non interactivity, a syntactical property, is thus equivalent to assimilating distinctness with doxastic independence, a semantical property.

\footnotetext{
${ }^{7}$ Normalization is not an issue in this definition, so the TBM conjunctive combination rule can be replaced by Dempster's rule of combination.
} 


\section{A.2 Belief on Evidential Structure}

An Evidential Structure [8] is defined as a triple $(\Omega, \mathcal{R}, \mathcal{E})$ where $(\Omega, \mathcal{R})$ is a propositional space (a set of propositions close under conjunctions and disjunctions and satisfying classical distributivity) and $\mathcal{E}$ is a set of couples $\left(\omega^{\prime}, \omega^{\prime \prime}\right) \in \Omega^{2}$ with $\omega^{\prime} \neq \omega^{\prime \prime}$ and $\omega^{\prime} \wedge \omega^{\prime \prime}={ }_{\epsilon} \perp$ where $\omega={ }_{\epsilon} \perp$ means that $\omega$ is a contradiction. Intuitively the couples of $\mathcal{E}$ are the pairs of conflictual propositions. Note that $\mathcal{R}$ is not a Boolean algebra (negation is not defined) but a distributive lattice.

The authors define bel as:

$$
\operatorname{bel}(X)=\sum_{\substack{X \wedge Z=Z: Z \in \mathcal{R} \\ \nexists Y \in \mathcal{R}, Y \neq \epsilon \perp, Y \wedge Z=Z, X \wedge Y=\epsilon \perp}} m(Z), \forall X \in \mathcal{R} .
$$

In the bomb counterexample (see section 3.4), we have the four propositions bomb, decoy, large, small where bomb $\wedge$ decoy $={ }_{\epsilon} \perp$ and large $\wedge$ small $={ }_{\epsilon} \perp$ (no fuzziness is involved here).

Suppose two categorical bbas $m_{1}($ large $\wedge$ bomb $)=1$ and $m_{2}($ small $\wedge$ bomb $)=1$. Thus bel $l_{1}($ bomb $)=1$ and bel $_{2}($ bomb $)=1$. Their combination produces $m_{12}($ large $\wedge$ small $\wedge$ bomb $)=1$, in which case bel $l_{12}($ bomb $)=0$ whereas many users would have like it to be 1 . Indeed both sources believe that 'the object is a bomb', and jointly they do not give it any support.

To show the result for bel $l_{12}($ bomb $)=0$, put $Y=$ decoy $\vee($ large $\wedge$ small $)$ in relation (24). It satisfies the exclusion requirements, hence the mass given to large $\wedge$ small $\wedge$ bomb is not included in bel $_{12}($ bomb $)$.

This, and the computational complexity, explain probably why this model was abandoned. Nevertheless, more recently and independently, beliefs on lattices were reintroduced in the DSm model [84].

\section{A.3 The combination rules}

We list the classical combination rules. We separate them according to the algebras on which their bbas are defined: the Boolean algebras or the distributive lattices. In the Boolean algebra case, we further separate the normalized rules that keep $m(\emptyset)=0$ from the unnormalized ones that allow $m(\emptyset)>0$. We list their definition in this section and we study some of their properties in section A.4.

\section{A.3.1 Normalized beliefs on Boolean algebras}

Generally belief functions are defined on the power set built from a frame of discernment $\Omega$, i.e. a set of mutually exclusive (and usually exhaustive) alternatives. We list many solutions proposed for the conjunctive combination of two bbas. In each one, $m$ denotes the bba obtained by the application of the corresponding combination rule. Each rule considers the term $k=\sum_{A \cap B=\emptyset} m_{1}(A) m_{2}(B)$, called the weight of conflict in [79], or the amount of conflict.

Dempster's rule of combination. [79] The most classical rule redistributes $k$ proportionally to each final bbm. It is applicable only if $k \neq 1$.

$$
m(X)= \begin{cases}\frac{1}{1-k} \sum_{A \cap B=X} m_{1}(A) m_{2}(B) & \forall X \subseteq \Omega, X \neq \emptyset \\ 0 & \text { if } X=\emptyset\end{cases}
$$

This operation is denoted $\oplus$ as in $m_{1} \oplus m_{2}$ or $m_{1 \oplus 2}$.

Yager's combination rule. $[112,113,49]$ The mass $k$ is transferred to $\Omega$.

$$
m(X)= \begin{cases}\sum_{A \cap B=X} m_{1}(A) m_{2}(B) & \forall X \subset \Omega, X \neq \Omega, X \neq \emptyset \\ m_{1}(\Omega) m_{2}(\Omega)+k, & \text { if } X=\Omega \\ 0 & \text { if } X=\emptyset\end{cases}
$$

Dubois and Prade's combination rule. [23] Whenever two focal elements have an empty intersection, the product of their masses is allocated to the union of the two focal elements.

$$
m(X)= \begin{cases}\sum_{A \cap B=X} m_{1}(A) m_{2}(B)+\sum_{\substack{A \cap B=\emptyset, A \cup B=X}} m_{1}(A) m_{2}(B), & \forall X \subseteq \Omega, X \neq \emptyset \\ 0 & \text { if } X=\emptyset\end{cases}
$$


Unified combination rule. [38, 52] These authors regroup many cases into a general family. The mass $k$ is distributed among the subsets of $\Omega$.

$$
m(X)= \begin{cases}\sum_{A \cap B=X} m_{1}(A) m_{2}(B)+w(X) k & \forall X \subseteq \Omega, X \neq \emptyset \\ 0 & \text { if } X=\emptyset\end{cases}
$$

where the coefficients satisfy $w(X) \geq 0$ and $\sum_{\emptyset \neq X \subseteq \Omega} w(X)=1$.

Weighted product combination rule. [118] The products of the masses are weighted by some measure $w(A, B)$ of intersection or set-agreement.

$$
m(X)=k^{*} \sum_{A \cap B=X} w(A, B) m_{1}(A) m_{2}(B) \quad \forall X \subseteq \Omega, X \neq \emptyset,
$$

where $k^{*}$ is a normalization factor so the sum of the masses is 1 . By construction $m(\emptyset)=0$. These rules are associative if $w(A \cap B, C)=w(A, B \cap C)$.

Among these rules, one finds:

- Zhang's center combination rule[118]

$$
m(X)=k^{*} \sum_{A \cap B=X} \frac{|X|}{|A| \cdot|B|} m_{1}(A) m_{2}(B) \quad \forall X \subseteq \Omega, X \neq \emptyset .
$$

This rule was also proposed by Fixsen-Mahler (who call it the Modified Dempster-Shafer Combination) [27], and by Sudano [107].

- Mahler : the conditioned Dempster-Shafer combination $B *_{\text {bel }} C[59]$

$$
m(X)=k^{*} \sum_{A \cap B=X} \frac{\operatorname{bel}(X)}{\operatorname{bel}(A) \operatorname{bel}(B)} m_{1}(A) m_{2}(B) \quad \forall X \subseteq \Omega, X \neq \emptyset,
$$

where bel is a third belief function also defined on $\Omega$.

In these rules, the ratios are 0 if the denominator is 0 . These two rules are associative, but not applicable when $k=1$.

See also $[106]$ for alternative suggestions.

Mixing combination rule. [64, 78] Bbas are averaged.

$$
m(X)=\sum_{i=1,2} w_{i} m_{i}(X) \quad \forall X \subseteq \Omega
$$

where $w_{i} \in[0,1]$ and $\sum_{i} w_{1}=1$. As the initial bbas $m_{i}$ are normalized, so is their average.

Weighted Average Operator (WAO). [42] In this particular form of unified combination rule, the conflict is transferred on the subsets proportionally to the mean mass given to the subsets.

$$
m(X)= \begin{cases}\sum_{Y \cap Z=X} m_{1}(Y) m_{2}(Z)+k\left(m_{1}(X)+m_{2}(X)\right) / 2 & \forall X \subseteq \Omega, X \neq \emptyset \\ k\left(m_{1}(\emptyset)+m_{2}(\emptyset)\right) / 2 & X=\emptyset\end{cases}
$$

An essentially similar rule is also described as the Proportional conflict redistribution rule (PCR) in [85]. In WAO, some mass can still be allocated to the empty set if $m_{1}$ or $m_{2}$ are not normalized, whereas $m(\emptyset)=0$ in the PCR rule.

Other proposals Many other rules have also been suggested. In [12, 14, 15], Daniel keeps track of the origin of each conflict. In [41], Josang develops a consensus operator described on binary frames, see also [42]. For other proposals, see $[4,117,108,83,57,56,54,43,10,107]$. Many proposed solutions are purely ad hoc. 


\section{A.3.2 Unnormalized beliefs on Boolean algebras}

This case corresponds to the TBM.

The TBM conjunctive combination rule. [104] The mass $k$ is allocated to the empty set. Thus:

$$
m(X)=\sum_{A \cap B=X} m_{1}(A) m_{2}(B) \quad \forall X \subseteq \Omega
$$

This operation is denoted (1) as in $m_{1} @ m_{2}$ or $m_{1} @ 2$. It generalizes the intersection operator to weighted sets. In the TBM literature, this rule is called the conjunctive combination rule, but we add the TBM label in this paper to avoid the confusion with the other rules. Note that this rule is at the core of almost all the rules described above (see relations (25), (26), (27), (28) and (31)).

\section{A.3.3 Beliefs on distributive lattices}

All these rules consider that the bbas are defined on Boolean algebras. Some authors do not require such a strict structure and replace it by a distributive lattice $[8,39,67,20,84,13,15]$. Comparison with bbas defined on Boolean algebras is delicate as the domain of the bbas becomes much larger (and complex).

This approach has the advantage that the components of the conflict are not just pooled together in a unique $\emptyset$ and cannot be individually recover.

Evidential structure. [8] Their definition of bel is unfortunately not satisfactory (see Appendix A.2).

Free DSm model. [84] The free DSm model is analogous to an evidential structure where the set $\mathcal{E}$ is empty (undefined) (see Appendix A.2). The distributive lattice is called the Dedekind's lattice $D^{\Omega}$. So by definition there is no conflict. As all bbas to be combined are normalized, the combination rule is syntactically the same as the TBM conjunctive combination rule (relation 32).

Hybrid DSm combination rule. [84] In the hybrid DSm model, conflicts are considered. Conflicts are introduced by a list of expressions like $A \in \emptyset$ where $\emptyset=\left\{\emptyset_{\mathcal{M}}, \emptyset\right\}$ is the set of all elements which have been forced by the user to be 'empty' through the constraints of the model, and $\emptyset$ is the classical empty set. Conceptually, the hybrid DSm model is analogous to the evidential structure where sets replace propositions and $\emptyset$ replaces $\mathcal{E}$.

These authors define $\operatorname{bel}(X)=\sum_{Y \subseteq X} m(Y)$. Nothing forbids a bba to give a mass 1 to $X \cap Y$ where $X, Y \in D^{\Omega}, X \cap Y \in \emptyset_{\mathcal{M}}$. In that case, $\operatorname{bel}(X)=1$ and $\operatorname{bel}(Y)=1$. So You put full belief in two sets that are in conflict. Is it adequate? Besnard's definition for bel (see Appendix A.2) was proposed just to avoid that behavior.

The proposed combination rule is similar to Dubois and Prade's combination rule (27), except for the case where some masses could be given to $\emptyset_{\mathcal{M}}$ in both $m_{i}$ 's. (a property not encountered in Dubois and Prade's model as they define their relation for Boolean lattices). The difference with Dubois and Prade rule lays in the term $S_{2}$ in relation (33). Unfortunately this term is a potential source of difficulties as the rule seems to be language dependent (see Appendix A.5).

$$
m(X)= \begin{cases}S_{1}(X)+S_{2}(X)+S_{3}(X) & \forall X \subseteq \Omega, X \notin \emptyset \\ 0, & \text { if } X \in \emptyset\end{cases}
$$


where:

$$
\begin{aligned}
& S_{1}(X)=\sum_{\substack{A, B \in D^{\Omega} \\
A \cap B=X}} m_{1}(A) m_{2}(B) \\
& S_{2}(X)= \sum_{\substack{A \in \emptyset, B \in \emptyset, \\
\text { if } X=\cup_{i \in \mathcal{U}(A, B)} \omega_{i} \text { and } \mathcal{U}(A, B) \notin \emptyset \\
\text { or } X=\Omega, \text { and } \mathcal{U}(A, B) \in \emptyset}} m_{1}(A) m_{2}(B) \\
& S_{3}(X)=\sum_{\substack{A, B \in D^{\Omega} A \cap B \in \emptyset, A \cup B=X}} m_{1}(A) m_{2}(B)
\end{aligned}
$$

where $D^{\Omega}$ is the Dedekind's lattice defined on the set $\Omega, \mathcal{U}(A, B)=u(A) \cup u(B)$ and $u(C)=\left\{\omega_{i}: \omega_{i}\right.$ is a singleton that composes $C$ \}.

\section{A.4 Comparing the rules}

Table 3 compares some properties of the combination rules listed in section A.3. It is restricted to static fusion (section 4). The criterion considered are the commutativity, the associativity, the resistance to refinement and some properties related to the combination with a categorical bbas.

Let $\otimes$ generically denote any of the analyzed conjunctive combination operators and let $m_{Z}$ denote a categorical bba focused on $Z$. Classically $m @ m_{Z}$ is understood as the conditioning of $m$ on $Z$, denoted $m[Z]$, and we will keep this name in the context of the other operators $\otimes$.

The criteria are defined as:

1. commutativity: $m_{1} \otimes m_{2}=m_{2} \otimes m_{1}$.

2. associativity: $\left(m_{1} \otimes m_{2}\right) \otimes m_{3}=m_{1} \otimes\left(m_{2} \otimes m_{3}\right)$

3. resistance to refinement: Let $\Omega^{*}$ be a refinement of $\Omega$ [79, page 114]. This criteria is satisfied when the result of the combination of the bbas after refinement on $\Omega^{*}$ is the refinement on $\Omega^{*}$ of the result of the combination of the initial bbas.

4. plausibility of false: $p l[Z](\bar{Z})=0$. This criteria reflect the idea that if You know that the actual value of $\Omega$ belongs to $Z$, the plausibility of $\bar{Z}$ should be null.

5. duplicate conditioning: $\left(m \otimes m_{Z}\right) \otimes m_{Z}=m \otimes m_{Z}$. Imagine You have some beliefs about the number of children of Paul. Learning for sure once or twice that Paul has less than three children should lead to the same revised belief.

6. iterated conditioning: $\left(m \otimes m_{Y}\right) \otimes m_{Z}=m \otimes m_{Y \cap Z}$ : In the last example, learning for sure that the number of children is less than five and then larger than three should induce the same revised belief as learning the number of children is less than five and larger than three.

7. conflictual conditioning: $\left(m_{Z} \otimes m_{\bar{Z}}\right) \otimes m$. We examine what is its value. It varies widely. Some rules conclude that there is a full conflict, some are not applicable leaving the user lost, some consider that $m$ holds as if the previous conflict between $m_{Z}$ and $m_{\bar{Z}}$ could be forgotten, some produce new bbas.

Table 3 presents a summary of the behavior of the rules presented in section A.3. A $\sqrt{ }$ means the criteria is satisfied, a - that it is not satisfied. Note that when we say that a rule fails to satisfy a property, we mean 'in general'. For instance, the mixing combination rules is commutative when $w_{1}=w_{2}=0.5$. Still we say it does not satisfy the commutativity, as it is so with other coefficients.

Note that once Yager's rule fails, Dubois and Prade's rule and of the unified combination rule fail too. Similarly once repeated conditioning fails, so does iterated conditioning.

The table shows that among the listed rules, Dempster's rule of combination, Mahler's rule and the TBM conjunctive combination rule satisfy all criteria. The problem with Mahler's rule used as a conjunctive combination rule comes from its dependence on some undefined external bel function. In fact Malher's intention was not to develop a full conjunctive combination rule, but to solve a problem related to Jeffrey's conditioning rule, an issue not tackled here $[40,80,37,93]$. The results of conflictual conditioning are not 


\begin{tabular}{|c|c|c|c|c|c|c|c|}
\hline & Commut & Assoc & $\begin{array}{c}\text { Resist } \\
\text { Refinem }\end{array}$ & $\begin{array}{l}\text { Plausib } \\
\text { of false }\end{array}$ & $\begin{array}{c}\text { Duplicate } \\
\text { condit }\end{array}$ & $\begin{array}{c}\text { Iterated } \\
\text { condit }\end{array}$ & $\begin{array}{l}\text { Conflictual } \\
\text { condit }\end{array}$ \\
\hline Dempster & $\sqrt{ }$ & $\sqrt{ }$ & $\sqrt{ }$ & $\sqrt{ }$ & $\sqrt{ }$ & $\sqrt{ }$ & inappl \\
\hline Yager & $\sqrt{ }$ & - & $\sqrt{ }$ & - & - & - & $m$ \\
\hline Dubois-Prade & $\sqrt{ }$ & - & $\sqrt{ }$ & - & - & - & $m$ \\
\hline Unified comb. & $\sqrt{ }$ & - & - & - & - & - & $w \otimes m$ \\
\hline Zhang & $\sqrt{ }$ & $\sqrt{ }$ & - & $\sqrt{ }$ & $\sqrt{ }$ & $\sqrt{ }$ & inappl \\
\hline Mahler & $\sqrt{ }$ & $\sqrt{ }$ & $\sqrt{ }$ & $\sqrt{ }$ & $\sqrt{ }$ & $\sqrt{ }$ & inappl \\
\hline Mixing comb. & - & - & $\sqrt{ }$ & - & - & - & $\left(w_{1} m_{Z}+w_{2} m_{\bar{Z}}\right) \otimes m$ \\
\hline WAO & $\sqrt{ }$ & - & $\sqrt{ }$ & - & - & - & $\left(0.5 m_{Z}+0.5 m_{\bar{Z}}\right) \otimes m$ \\
\hline TBM & $\sqrt{ }$ & $\sqrt{ }$ & $\sqrt{ }$ & $\sqrt{ }$ & $\sqrt{ }$ & $\sqrt{ }$ & confl \\
\hline
\end{tabular}

Table 3: Comparison of the properties listed in section A.4 for the combination rules on Boolean algebras listed in section A.3.

as simple. Inapplicability is evidently embarrassing for the user. That the end result is $m$ is amazing as it just mean we treat the conflictual data as if they did not exist. The TBM solution mentions the existence of the conflict and sticks to it what is also embarrassing for the user but reflects the actual situation. At least it will not crash the program as it could easily be the case with the inapplicable answer ${ }^{8}$. The other three solutions are difficult to justify. To decide which is the best solution is a matter of personal opinion.

For what concerns the Free DSm model, the rule satisfies syntactically the same properties as the TBM conjunctive combination rule, and the Hybrid DSm combination rules does not satisfy the rules unsatisfied by Dubois and Prade's rule.

\section{A.5 Language dependency and the Hybrid DSm rule}

We illustrate the issue about the language dependency of the hybrid DSm rule of combination presented by relation (33).

Suppose the next six propositions : $c=$ ' $\mathrm{X}$ is a car', $d=$ 'X is driven by a chauffeur', $\bar{d}=$ ' $\mathrm{X}$ is not driven by a chauffeur', $y=$ ' $\mathrm{X}$ is yellow', $\bar{y}=$ ' $\mathrm{X}$ is not yellow', $u={ }^{\prime} \mathrm{X}$ is a utility vehicle'. The idea is that a car driven by a chauffeur is a taxi and a yellow car is also a taxi. Besides a taxi is not a utility vehicle, a utility vehicle can be a car (like a Jeep) or driven by a chauffeur (like an ambulance) but never both assuming utility vehicles that are cars are always driven by the user itself. Identically a utility vehicle can be yellow, but not if it is a car as it would create confusion for users.

The propositions $c, d, y, u, \bar{y}, \bar{d}$ are the six propositions of the distributive lattice used in the DSmT, where $d \cap \bar{d} \in \emptyset, y \cap \bar{y} \in \emptyset, c \cap d \cap u \in \emptyset, c \cap y \cap u \in \emptyset$ as well as all their intersections.

The concept of equivalence is not defined in DSm model. So we cannot express directly that $c \cap y \equiv c \cap d$. We can nevertheless write $c \cap y \cap \bar{d} \in \emptyset$ and $c \cap \bar{y} \cap d \in \emptyset$ which together translate the idea expressed by the equivalence, and we thus add these two elements in $\emptyset$.

In table 4, we present two sources that share the same elementary propositions, the same lattice and the same $\emptyset$.

Our critique against the $S_{2}$ term in the Hybrid DSm rule (relation 35) is based on the doxastic equivalence. In the Boolean algebra case, two propositions $p_{1}$ and $p_{2}$ are doxastically equivalent if they are equivalent given what You accept as true (called the evidential corpus and denoted $E C_{\text {You }}$ ), i.e., $p_{1} \wedge E C_{\text {You }} \equiv p_{2} \wedge E C_{\text {You }}$ [96].

The doxastic consistency principle states that two doxastically equivalent propositions must receive the same degree of belief [96].

In the case of distributive lattice, equivalence does not exist, so we rewrite it as: two propositions $p_{1}$ and $p_{2}$ are doxastically equivalent given $E C_{\text {You }}$ if there exist propositions $e, f, g_{1}, g_{2}, \bar{g}_{1}, \bar{g}_{2}$ with $p_{i}=e \cap f \cap g_{i}, i=1,2$, and

- $f \cap g_{1} \cap E C_{\text {You }} \notin \emptyset$,

${ }^{8}$ Note that in the classical programs for belief propagation, like the VBS [81] as implemented in TBMLAB, normalization is not performed, except when displaying the data, so the VBS resists against such a crash... but in fact it works with the TBM conjunctive combination rule. 
- $f \cap g_{2} \cap E C_{\text {You }} \notin \emptyset$,

- $f \cap g_{1} \cap \bar{g}_{2} \cap E C_{\text {You }} \in \emptyset$,

- $f \cap \bar{g}_{1} \cap g_{2} \cap E C_{\text {You }} \in \emptyset$,

- for $i=1,2, \forall x, f \cap E C_{\text {You }} \cap\left(g_{i} \cup \bar{g}_{i} \cup x\right)=f \cap E C_{\text {You }} \cap\left(g_{i} \cup \bar{g}_{i}\right)$.

It means that $g_{1}$ and $g_{2}$, non conflictual with $f \cap E C_{\text {You }}$, are equivalent in context where $f \cap E C_{\text {You }}$ holds even if $e$ and $f$ are conflictual. The last requirement means that, for $i=1,2, g_{i}$ and $\bar{g}_{i}$ are 'exhaustive' in context where $f \cap E C_{\text {You holds. }}$

In our example, both Source 1 and Source 2 accept the same information as true (the information in the upper part of table 4). We denote it as $E C$.

We show now that $c \cap d \cap u$ and $c \cap y \cap u$ are doxastically equivalent under $E C$. Replace $u$ for $e, c$ for $f$, $d$ for $g_{1}$ and $y$ for $g_{2}$ and we get

- $c \cap d \cap E C \notin \emptyset$,

- $c \cap y \cap E C \notin \emptyset$,

- $c \cap d \cap \bar{y} \cap E C \in \emptyset$,

- $c \cap \bar{d} \cap y \cap E C \in \emptyset$,

- $c \cap(d \cup \bar{d})=c$,

- $c \cap(y \cup \bar{y})=c$.

As far as $c \cap y \cap u$ and $c \cap d \cap u$ are doxastically equivalent for Source 1, the doxastic consistency principle implies that for Source 1 , $\operatorname{bel}_{1 j}(c \cap y \cap u)=\operatorname{bel}_{1 j}(c \cap d \cap u)=1$ for $\mathrm{j}=1,2$. The symmetrical can be done for Source 2. Hence both sources share the same beliefs. The only difference is that one uses $c \cap d$ whereas the other uses $c \cap y$ to describe a taxi.

Nevertheless when we apply the Hybrid DSm combination rule on the data of table 4, the resulting $m_{1,12}$ and $m_{2,12}$ are not equal. For instance they do not give the same belief to $c \cup d \cup u$. One would have expected that if two sources share the same information and the same beliefs, the combination of their beliefs should also be equal.

So something is wrong in the system as described. We must:

- adapt the doxastic consistency principle, or

- acknowledge that the bbas $m_{i 1}$ and $m_{i 2}$ of table 4 do not correctly translate the constraints in $\emptyset$, a concept that seems undefined in DSm model, and all four must be changed into $m(c \cap d \cap y \cap u)=1$, or

- adapt the rule in order to take into account that $c \cap d=c \cap y=c \cap d \cap y$ in order to reallocate the $m_{i j}$ to the some focal element, or

- accept that the $S_{2}$ of the Hybrid DSm combination rule is language dependent.

It is our opinion that the Hybrid DSm combination rule as presented by its authors seems to need further specifications in order to resolve the present issue.

\section{A.6 Stepwise elimination of conflictual bbas}

To perform the stepwise elimination of the bbas that contradict the conclusions reached by the remaining bbas, the next equations are useful [3].

Suppose $n+1$ bbas $m_{0}, m_{1}, \ldots, m_{n}$. Let $m_{1 \ldots n}=m_{1} @ m_{2} \ldots @ m_{n}$, and $m_{0 \ldots n}=m_{0} @ m_{1 \ldots n}$.

After elimination of the basic belief assignment $m_{0}$ from $m_{0 \ldots n}$ the mass given by the remaining bba to $\emptyset$ is given by:

$$
m_{1 \ldots n}(\emptyset)=\sum_{A \subseteq \Omega} c(A) m_{0 \ldots n}(A)
$$

where

$$
c(A)= \begin{cases}\sum_{X \subseteq A} \frac{(-1)^{|X|}}{q_{0}(X)} & \text { iff } q(A) \neq 0 \\ 0 & \text { iff } q(A)=0 .\end{cases}
$$




\begin{tabular}{|c|c|c|}
\hline & Source 1 & Source 2 \\
\hline language & \multicolumn{2}{|c|}{$\begin{array}{c}c, u, y, \bar{y} \\
\quad d, \bar{d}\end{array}$} \\
\hline $\bar{\emptyset}$ & \multicolumn{2}{|c|}{$\begin{array}{c}d \cap \bar{d}, y \cap \bar{y} \\
c \cap d \cap u, c \cap y \cap u \\
c \cap y \cap \bar{d}, c \cap \bar{y} \cap d \\
c \cap(y \cup \bar{y})=c, c \cap(d \cup \bar{d})=c\end{array}$} \\
\hline $\begin{array}{c}\text { bba } m_{i 1} \\
\text { bba } m_{i 2} \\
\text { Hybrid DSm Rule } \\
\text { belief }\end{array}$ & $\begin{array}{c}m_{1,1}(c \cap d \cap u)=1 \\
m_{1,2}(c \cap d \cap u)=1 \\
m_{1,12}(c \cup d \cup u)=1 \\
b l_{1,12}(c \cup d \cup u)=1\end{array}$ & $\begin{array}{c}m_{2,1}(c \cap y \cap u)=1 \\
m_{2,2}(c \cap y \cap u)=1 \\
m_{2,12}(c \cup y \cup u)=1 \\
b e l_{2,12}(c \cup d \cup u)=0\end{array}$ \\
\hline
\end{tabular}

Table 4: Example where the component $S_{2}$ of the Hybrid DSm combination rule depends on the language.

and $q$ is the commonality function.

The coefficients $c(A)$ can be computed by the same Möbius transform that links the implicability function $b$ function to the bba $m$, replacing $b(X)$ by $1 / q(X)$ and multiplying the resulting terms by $(-1)^{|X|}[46,45,99]$.

We can judge how much the conflict would be reduced by eliminating from the combination $m_{0 \ldots n}$ the belief function $m_{i}, i=0,1, \ldots, n$, , by computing $m_{1 \ldots i-1, i+1, \ldots n}(\emptyset)$ using the elimination formula (37). The bba to be eliminated is the one for which this term is the smallest. The procedure can then be iterated.

Acknowledgment. The author thanks Wu Li, Weiru Liu, Philippe Besnard and Florentin Smarandache for the interesting discussions we had about this article. Special thanks to Milan Daniel for his efficient help. We also thank the referees who push us to clarify issues that we felt - erroneously - as obvious.

\section{References}

[1] J. Aczel. Lectures on Functional Equations and their Applications. Academic Press, New York, NY, 1966.

[2] A. Appriou. Probabilités et incertitude en fusion de données multi-senseurs. Revue Scientifique et Technique de la Défense, 11:27-40, 1991.

[3] A. Ayoun and Ph. Smets. Data association in multi-target detection using the transferable belief model. Inter. J. Intell. Systems, 16:1167-1182, 2001.

[4] J. F. Baldwin, T. P. Martin, and B. W. Pilsworth. FRIL-fuzzy and evidential reasoning in artificial intelligence. Research Studies Press, Tauton, Somerset, England, 1995.

[5] B. Ben Yaghlane, Ph. Smets, and K. Mellouli. Belief function independence: I. the marginal case. Int. J. Approximate Reasoning, 29:47-70, 2001.

[6] B. Ben Yaghlane, Ph. Smets, and K. Mellouli. Belief function independence: II. the conditional case. Int. J. Approximate Reasoning, 31:31-75, 2002.

[7] S. Benferhat, A. Saffiotti, and Ph. Smets. Belief functions and default reasoning. Artificial Intelligence, 122:1-69, 2000.

[8] P. Besnard, P. Jaouen, and J. Ph. Perin. Extending the transferable belief model for inconsistency handling. In IPMU-96, editor, Information Processing and Management of Uncertainty, pages 143-148, 1996.

[9] John Cantwell, Johan Schubert, and Johan Walter. Conflict-based force aggregation. In Proceedings of the Sixth International Command and Control Research and Technology Symposium, pages Track 7, Paper 031,1-15, Washington, 2001. US Department of Defence CCRP. 
[10] M. Cattaneo. Combining belief functions issued from dependent sources. In 3rd International Symposium on Imprecise Probabilities and Their Applications ISIPTA03, 2003.

[11] B. R. Cobb and P. P. Shenoy. A comparison of methods for transforming belief function models to probability models. In T. D. Nielsen and N. L. Zhang, editors, Symbolic and Quantitative Approaches to Reasoning with Uncertainty, pages 255-266. Springer-Verlag, Berlin, 2003.

[12] M. Daniel. Contradiction in combination of belief functions. In IPMU-98, editor, Information Processing and Management of Uncertainty, pages 56-63, 1998.

[13] M. Daniel. Associativity and contradiction in combination of belief functions. In IPMU-2000, editor, Information Processing and Management of Uncertainty, pages 133-140, 2000.

[14] M. Daniel. Distribution of contradictive belief masses in combination of belief functions. In B. BouchonMeunier, R. R. Yager, and L. A. Zadeh, editors, Information, Uncertainty and Fusion, pages 431-446. Kluwer Acad. Press, Boston, 2000.

[15] M. Daniel. Associativity in combination of belief functions; a derivation of minC combination. Soft Computing, 7:288-296, 2003.

[16] F. Delmotte and P. Smets. Target identification based on the transferable belief model interpretation of Dempster-Shafer model. IEEE Transactions on Systems, Man and Cybernetics, A 34:457-471, 2004.

[17] A. P. Dempster. Upper and lower probabilities induced by a multiple valued mapping. Ann. Math. Statistics, 38:325-339, 1967.

[18] A. P. Dempster. A generalization of Bayesian inference. J. Roy. Statist. Soc. B., 30:205-247, 1968.

[19] T. Denoeux and M.-H. Masson. Evclus: evidential clustering of proximity data. IEEE Trans. Systems, Man and Cybernetics: B, 34:95-109, 2004.

[20] J. Dezert. Foundations for a new theory of plausible and paradoxical reasoning. Information and Security, 9:13-57, 2002.

[21] D. Dubois, P. Garbolino, H.E. Kyburg, H. Prade, and Ph. Smets. Quantified uncertainty. J. Applied Non-Classical Logics, 1:105-197, 1991.

[22] D. Dubois and H. Prade. On the unicity of Dempster rule of combination. International Journal of Intelligent System, 1:133-142, 1986.

[23] D. Dubois and H. Prade. A set-theoretic view of belief functions: logical operations and approximations by fuzzy sets. International Journal of General Systems, 12:193-226, 1986.

[24] D. Dubois, M. P. Wellman, B. D'Ambrosio, and Ph. Smets, editors. Uncertainty in Artificial Intelligence 92. Morgan Kaufman, San Mateo, Ca, 1992.

[25] S. Fabre. Apport de l'information contextuelle à la fusion multicapteurs. Ph.d. thesis, Ecole Normale Spuérieure de l'Aéropautique et de l'Espace, 1999.

[26] S. Fabre, A. Appriou, and X. Briottet. Presentation and description of two classification methods using data fusion on sensor management. Information Fusion, 2:49-71, 2001.

[27] D. Fixsen and R. P. S. Mahler. The modified Dempster-Shafer approach to classification. IEEE-SMC, A, 27:96-104, 1997.

[28] Fusion00, editor. Proceedings of the Third International Conference on Information Fusion, Paris, France, 2000.

[29] Fusion03, editor. Proceedings of the Sixth International Conference on Information Fusion, Cairns, Australia, 2003.

[30] D. M. Gabbay and Ph. Smets, editors. Handbook of Defeasible Reasoning and Uncertainty Management Systems, Vol. 1, volume 1. Kluwer, Doordrecht, The Netherlands, 1998.

[31] D. Gruyer, C. Royere, and V. Cherfaoui. Heterogeneous multi-criteria combination with partial or full information. In Fusion03 [29], pages 1-1.

[32] R. Haenni. Are alternatives to Dempster's rule of combination alternatives? Int. J. Information Fusion, 3:237-241, 2002. 
[33] R. Haenni. Shedding new light on Zadeh's criticism of Dempster's rule of combination. submitted, 2005.

[34] R. Haenni, J. Kohlas, and N. Lehmann. Probabilistic argumentation systems. In D. M. Gabbay and $\mathrm{Ph}$. Smets, editors, Handbook of Defeasible Reasoning and Uncertainty Management Systems, volume 5, pages 221-288. Kluwer, Doordrecht, The Netherlands, 2000.

[35] P. Hajek. Deriving Dempsters rule. In IPMU-92, editor, Information Processing and Management of Uncertainty, pages 73-75, 1992.

[36] Y. T. Hsia. Characterizing belief with minimum commitment. In IJCAI-91, editor, Int. Joint Conf. on Artificial Intelligence, pages 1184-1189. Morgan Kaufman, San Mateo, Ca, 1991.

[37] H. Ichihashi and H. Tanaka. Jeffrey-like rules of conditioning for the Dempster-Shafer theory of evidence. Inter. J. Approximate Reasoning, 3:143-156, 1989.

[38] T. Inagaki. Interdependence between safety-control policy and multiple-sensor schemes via DempsterShafer theory. IEEE Trans. on Reliability, 40:182-188, 1991.

[39] P. Jaouen. Modélisation de contradictions par extension de la théorie de l'évidence. Ph.d. thesis, Université de Rennes 1, 1997.

[40] R. C. Jeffrey. The Logic of Decision. 2nd Ed. Univ. Chicago Press, Chicago, 1983.

[41] A. Josang. The consensus operator for combining beliefs. Artificial Intelligence Journal, 142:157-170, 2002.

[42] A. Josang, M. Daniel, and P. Vannoorenberghe. Strategies for combining conflicting dogmatic beliefs. In Fusion03 [29], pages 1133-1140.

[43] A. V. Joshi, S. C. Sahasrabudhe, and K. Shankar. Sensitivity of combination schemes under conflicting conditions and a new method. In Jacques Wainer and Ariadne Carvalho, editors, Advances in Artificial Intelligence, 12th Brazilian Symposium on Artificial Intelligence, SBIA '95, volume 991 of Lecture Notes in Computer Science, pages 39-48. Springer, 1995.

[44] A. Kaufmann and G. Boulaye. Theorie des treillis en vue des applications. Masson, Paris, 1978.

[45] R. Kennes. Computational aspects of the Möbius transform of a graph. IEEE-SMC, 22:201-223, 1992.

[46] R. Kennes and Ph. Smets. Computational aspects of the Möbius transform. In P. P. Bonissone, M. Henrion, L. N. Kanal, and J. F. Lemmer, editors, Uncertainty in Artificial Intelligence 6, pages 401-416. North Holland, Amsterdam, 1991.

[47] F. Klawonn and E. Schwecke. On the axiomatic justification of Dempster's rule combination. International Journal of Intelligent Systems, 7:469-478, 1992.

[48] F. Klawonn and $\mathrm{Ph}$. Smets. The dynamic of belief in the transferable belief model and specialization - generalization matrices. In Dubois et al. [24], pages 130-137.

[49] J. Kohlas. The reliability of reasoning with unreliable arguments. Ann. Op. Res., 32:76-113, 1991.

[50] J. Kohlas and P. A. Monney. A Mathematical Theory of Hints: An Approach to Dempster-Shafer Theory of Evidence. Lecture Notes in Economics and Mathematical Systems No. 425. Springer-Verlag, 1995.

[51] R. Kruse and E. Schwecke. Specialization: a new concept for uncertainty handling with belief functions. Int. J. Gen. Systems, 18:49-60, 1990.

[52] E. Lefèvre, O. Colot, and P. Vannoorenberghe. Belief function combination and conflict managment. Information fusion, 3:149-162, 2002.

[53] D. Lewis. Probabilities of conditionals and conditional probabilities. Philosophical Review, 85:297-315, 1976.

[54] X. N. Ling and W. G. Rudd. Combining opinions from several experts. Applied Artificial Inteligence, 3:439-452, 1989.

[55] W. Liu. Analysing the degree of conflict among belief functions. Personnal communincation, 2004. 
[56] W. Liu and A. Bundy. The combination of different pieces of evidence using incidence calculus. Technical report, Department of Artificial Intelligence, University of Edinburg, 1992.

[57] W. Liu and J. Hong. Re-investigating Dempster's idea on evidence combination. Knowledge and Information Systems, 2:223-241, 2000.

[58] R. P. S. Mahler. Combining ambiguous evidence with respect to ambiguous a priori knowledge, ii: Fuzzy logic. Fuzzy Sets ans Systems, 75:319-354, 1995.

[59] R. P. S. Mahler. Combining ambiguous evidence with respect to ambiguous a priori knowledge, i: Boolean logic. IEEE-SMC, A, 26:27-41, 1996.

[60] N. Milisavljevic. Analysis and Fusion Using Belief Functions Theory of Multisensor Data for CloseRange Humanitarian Mine Detection. Ph.d. thesis, Ecole Normale Supérieure de Télécomminications, Paris, France, 2001

[61] N. Milisavljevic, I. Bloch, and M. Acheroy. Characterization of mine detection sensors in terms of belief function and their fusion. In Fusion00 [28], pages ThC3.15-ThC3.22.

[62] N. Milisavljevic, I. Bloch, and M. Acheroy. Modeling, combining and discounting mine detection sensors within Dempster-Shafer framework. In Detection Technologies for Mines and Minelike Targets, volume 4038, pages 1461-1472. SPIE Press, Orlando, USA, 2000.

[63] N. Milisavljevic, S. van den Broek, I. Bloch, P. B. W. Schwering, H. A. Lensen, and M. Acheroy. Comparison of belief function and voting method for fusion of mine detection sensors. In Detection and Remediation Technologies for Mines and Minelike Targets VI, volume 4394. SPIE Press, Orlando, USA, 2001.

[64] C. K. Murphy. Combining belief functions when evidence conflicts. Decision Support Systems, 29:1-9, 2000.

[65] H. T. Nguyen. On random sets and belief functions. J. Math.Anal. Applic., 65:531-542, 1978.

[66] H. T. Nguyen and Ph. Smets. On dynamics of cautious belief and conditional objects. Int. J. Approximate Reasoning, 8:89-104, 1993.

[67] J.-P. Perin. Théorie de l'évidence: modélisation et application pour un SIC. Ph.d. thesis, Université de Rennes 1, 1997.

[68] B. Ristic and Ph. Smets. Recursive classification of multiple objects using discordant and non-specific data. sumitted, 2004.

[69] C. Royere, D. Gruyer, and V. Cherfaoui. Data association within belief theory. In Fusion00 [28], pages TuD2-9.

[70] J. Schubert. On nonspecific evidence. Int. J. Intell. Syst., 8(6):711-725, 1993.

[71] J. Schubert. Cluster-based Specification Techniques in Dempster-Shafer Theory for an Evidential Intelligence Analysis of Multiple Target Tracks. Ph.d. thesis, Royal Institute of Technology, Stockholm, 1994.

[72] J. Schubert. Cluster-based specification techniques in Dempster-Shafer theory. In C. Froidevaux and J. Kohlas, editors, Symbolic and Quantitative Approaches to Uncertainty, pages 395-404. SpringerVerlag, Berlin, 1995.

[73] J. Schubert. Fast Dempster-Shafer clustering using a neural network structure. In B. Bouchon-Meunier, R. R. Yager, and L. A. Zadeh, editors, Information, Uncertainty and Fusion, pages 419-430. Kluwer, Boston, MA, 1999.

[74] J. Schubert. Managing inconsistent intelligence. In Fusion00 [28], pages TuB4/10-16.

[75] J. Schubert. Clustering belief functions based on attracting and conflicting metalevel evidence. In IPMU-2002, editor, Information Processing and Management of Uncertainty, pages 571-578,, 2002.

[76] J. Schubert. Evidential force aggregation. In Fusion03 [29], pages 1223-1229.

[77] J. Schubert. Clustering belief functions based on attracting and conflicting metalevel evidence using Potts spin mean field theory. Information Fusion, forthcoming, 2004. 
[78] K. Sentz and S. Ferson. Combination of evidence in Dempster-Shafer Theory. Technical report, SANDIA Tech. Report, SAND2002-0835, 2002.

[79] G. Shafer. A Mathematical Theory of Evidence. Princeton Univ. Press. Princeton, NJ, 1976.

[80] G. Shafer. Jeffrey's rule of conditioning. Philisophy of Sciences, 48:337-362, 1981.

[81] G. Shafer, P. P. Shenoy, and K. Mellouli. Propagating belief functions in qualitative Markov trees. Int. J. Approximate Reasoning, 1:349-400, 1987.

[82] G. Shafer and A. Tversky. Languages and designs for probability. Cognitive Sc., 9:309-339, 1985.

[83] S. Shi, M. E. C. Hull, and D. A. Bell. A new rule for updating evidence. In Z.W. Ras and M. Zemankova, editors, Methodologies for Intelligent Systems, 8th International Symposium, ISMIS '94, pages 95-104. Springer, 1994.

[84] F. Smarandache and J. Dezert. Advances and applications of DSmT for information fusion. American Research Press, Rehoboth, NM., 2004.

[85] F. Smarandache and J. Dezert. Proportional conflict redistribution rule. Personnal communication, 2004.

[86] Ph. Smets. Un modèle mathématico-statistique simulant le processus du diagnostic médical. Ph.d. thesis, Université Libre de Bruxelles, (available through University Microfilm International, 30-32 Mortimer street, London W1N 7RA, thesis 80-70,003), 1978.

[87] Ph. Smets. Belief functions. In Ph. Smets, E. H. Mamdani, D. Dubois, and H. Prade, editors, Non Standard Logics for Automated Reasoning, pages 253-286. Academic Press, London, 1988.

[88] Ph. Smets. The combination of evidence in the transferable belief model. IEEE Pattern Analysis and Machine Intelligence, 12:447-458, 1990.

[89] Ph. Smets. Constructing the pignistic probability function in a context of uncertainty. In M. Henrion, R. D. Shachter, L. N. Kanal, and J. F. Lemmer, editors, Uncertainty in Artificial Intelligence 5, pages 29-40. North Holland, Amsterdam, 1990.

[90] $\mathrm{Ph}$. Smets. The nature of the unnormalized beliefs encountered in the transferable belief model. In Dubois et al. [24], pages 292-297.

[91] Ph. Smets. The transferable belief model and random sets. Int. J. Intell. Systems, 7:37-46, 1992.

[92] Ph. Smets. Belief functions: the disjunctive rule of combination and the generalized Bayesian theorem. Int. J. Approximate Reasoning, 9:1-35, 1993.

[93] Ph. Smets. Jeffreys rule of conditioning generalized to belief functions. In D. Heckerman and A. Mamdani, editors, Uncertainty in Artificial Intelligence 93, pages 500-505. Morgan Kaufmann, San Mateo, Ca, USA, 1993.

[94] Ph. Smets. What is Dempster-Shafer's model? In Yager et al. [111], pages 5-34.

[95] Ph. Smets. The alpha-junctions: combination operators applicable to belief functions. In D.M Gabbay, R. Kruse, A. Nonnengart, and H.J. Ohlbach, editors, Qualitative and quantitative practical reasoning, pages 131-153. Springer, 1997.

[96] Ph. Smets. The normative representation of quantified beliefs by belief functions. Artificial Intelligence, 92:229-242, 1997.

[97] Ph. Smets. Probability, possibility, belief: Which and where? In Gabbay and Smets [30], pages 1-24.

[98] Ph. Smets. The transferable belief model for quantified belief representation. In Gabbay and Smets [30], pages 267-301.

[99] $\mathrm{Ph}$. Smets. The application of the matrix calculus to belief functions. Int. J. Approximate Reasoning, 31:1-30, 2002.

[100] Ph. Smets. Decision making in a context where uncertainty is represented by belief functions. In R. P. Srivastava and T.J. Mock, editors, Belief Functions in Business Decisions, pages 17-61. PhysicaVerlag, Heidelberg, Germany, 2002. 
[101] Ph. Smets. Belief functions on real numbers. Submitted: see http://iridia.ulb.ac.be/ psmets, 2004.

[102] Ph. Smets. Decision making in the TBM: the necessity of the pignistic transformation. Int. J. Approximate Reasoning, 38:133-147, 2005.

[103] Ph. Smets. Managing deceitful reports with the Transferable Belief Model. In submitted, 2005.

[104] Ph. Smets and R. Kennes. The transferable belief model. Artificial Intelligence, 66:191-234, 1994.

[105] Lea Sombe. A glance at revision and updating in knowledge bases. Inter. J. Intelligent Systems, 9:1-28, 1994.

[106] J. J. Sudano. The system probability information content (PIC) relationship to contributing components, combining independent multi-sources beliefs, hybrid and pedigree pignistic probabilities. In Fusion02, editor, Proceedings of the Fifth International Conference on Information Fusion, volume 2, pages 1277-1283, Annapolis, MD, USA, 2002.

[107] J. J. Sudano. A generalized belief fusion algorithm. In Fusion03 [29], pages 1126-1132.

[108] B. Tonn. An algorithmic approach to combining belief functions. International Journal of Intelligent Systems, 11:463-476, 1996.

[109] R. R. Yager. Entropy and specificity in a mathematical theory of evidence. International Journal of General Systems, 9(4):249-260, 1983.

[110] R. R. Yager. Hedging in the combination of evidence. Journal of Information and Optimization Science, 4:73-81, 1983.

[111] R. R. Yager, J. Kacprzyk, and M. Fedrizzi, editors. Advances in the Dempster-Shafer Theory of Evidence. Wiley, New York, 1994.

[112] R.R. Yager. On the Dempster-Shafer framework and new combination rules. Information Sciences, 41:93-137, 1987.

[113] R.R. Yager. Quasi-associative operations in the combination of evidence. Kybernetes, 16:37-41, 1987.

[114] L. A. Zadeh. On the validity of Dempster's rule of combination of evidence. Technical Report UCB/ERL M79/24, University of California, Berkely, 1979.

[115] L. A. Zadeh. A mathematical theory of evidence (book review). AI Magazine, 5:81-83, 1984.

[116] L. A. Zadeh. A simple view of the Dempster-Shafer theory of evidence and its implication for the rule of combination. AI Magazine, 7:85-90, 1986.

[117] B. P. Zeigler. Some properties of modified Dempster-Shafer operators in rule based inference systems. Int. J. General Systems, 14:345-356, 1988.

[118] L. Zhang. Representation, independence, and combination of evidence in the Dempster-Shafer theory. In Yager et al. [111], pages 51-69. 


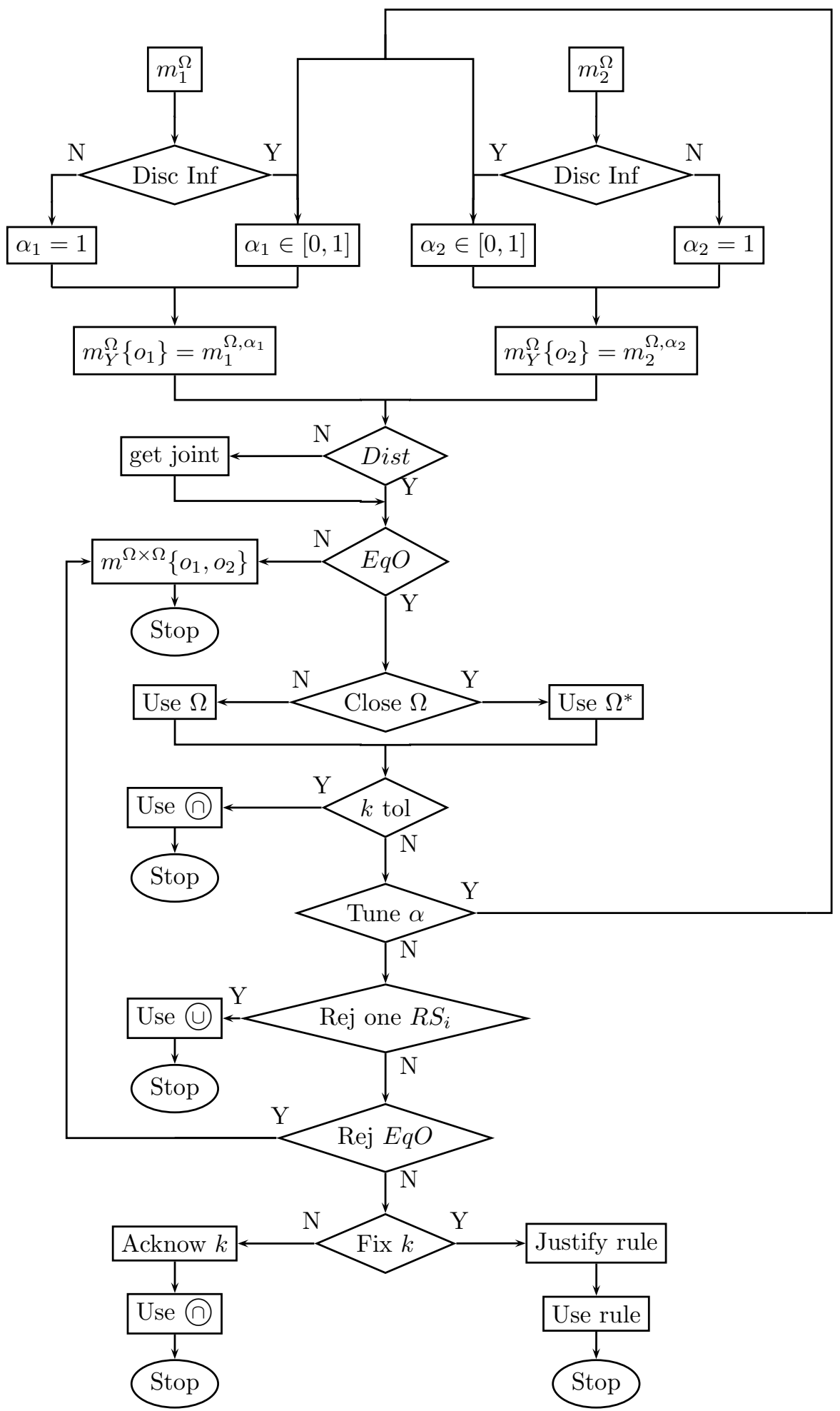

Figure 1: Flowchart of a possible expert systems for handling conflict. 